\title{
Bio-Inspired Trailing Edge Noise Control
}

lan Clark, Nathan Alexander, and William Devenport, Virginia Tech Stewart Glegg, Florida Atlantic University, Justin Jaworski, Lehigh University Nigel Peake and Conor Daly, Cambridge University 


\section{Motivation}

Wind turbines are regulated for noise which limits their size, location, and operation.

A significant percentage of wind turbines are de-rated to comply with these regulations.

This results in a loss of Annual Energy Production for each decibel of noise reduction required.

We seek to reduce or eliminate the dominant noise source of wind turbines, which is trailing edge noise at the outer portion of the blades (where the most power is produced). 


\section{Inspiration}

Certain species of owl that fly silently above $1.5 \mathrm{kHz}$ have down-like hairs on their feathers.

These hairs tend to form a canopy suspended over the surface of the feather.

A similar structure has been shown to attenuate pressure fluctuations at the underlying surface.
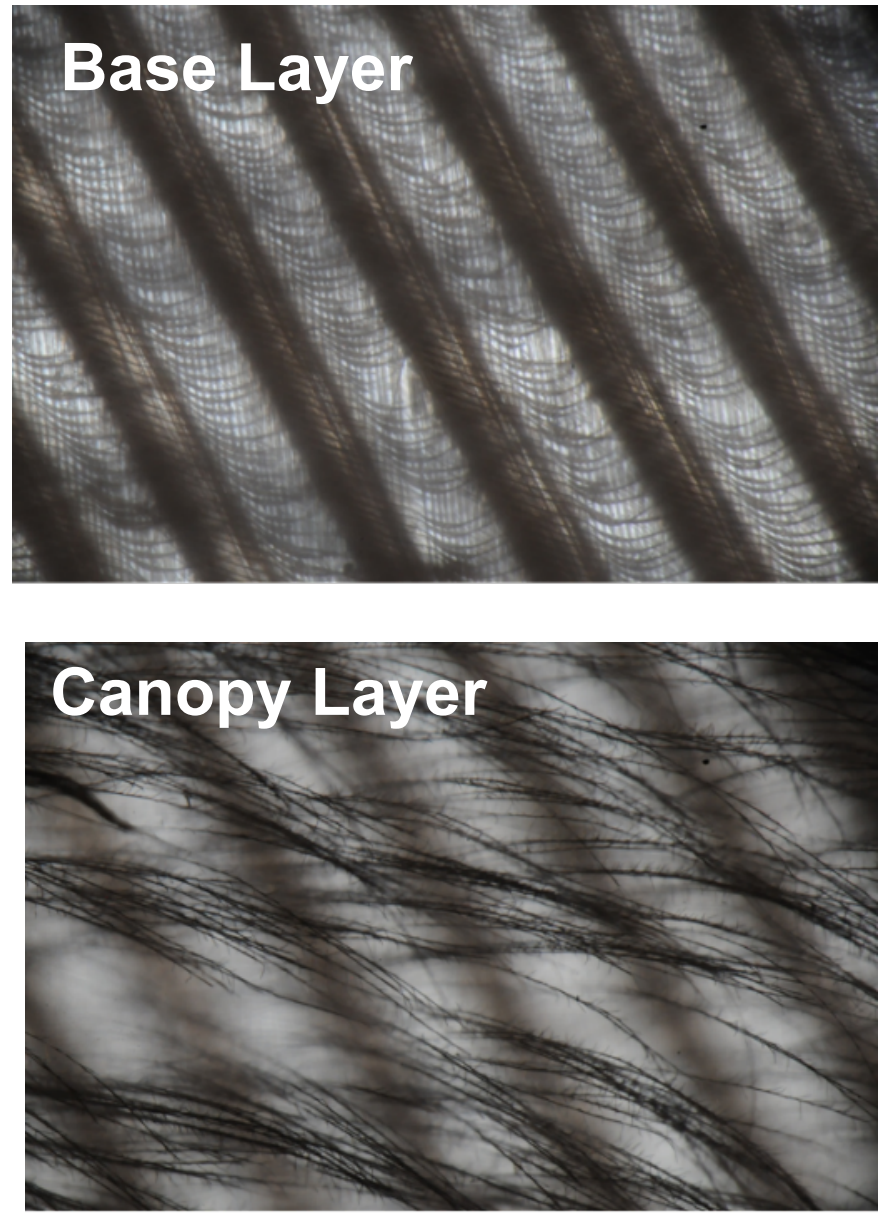

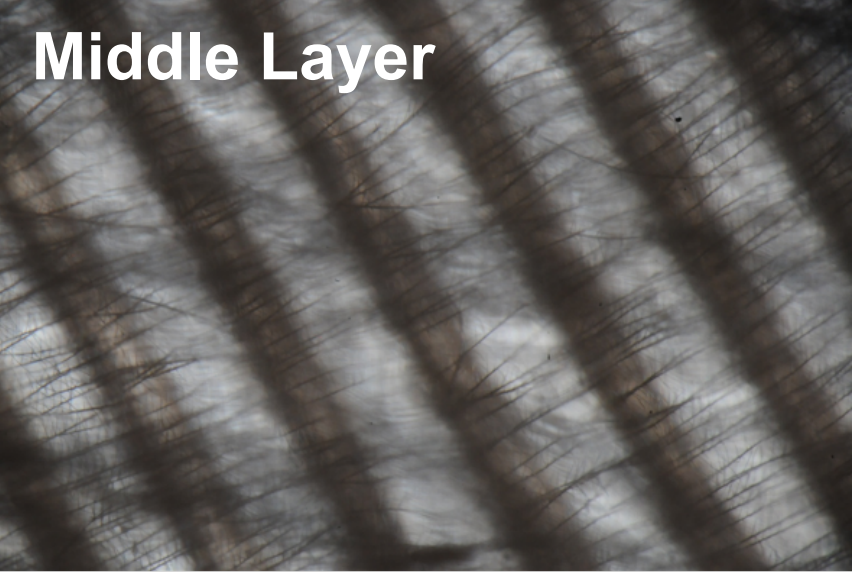

Canopy height $\cong 0.5 \mathrm{~mm}$ Individual hair $\mathrm{Re} \cong 7$ Canopy open area ratio $\cong 70 \%$ 


\section{Unidirectional Fabric Canopy}

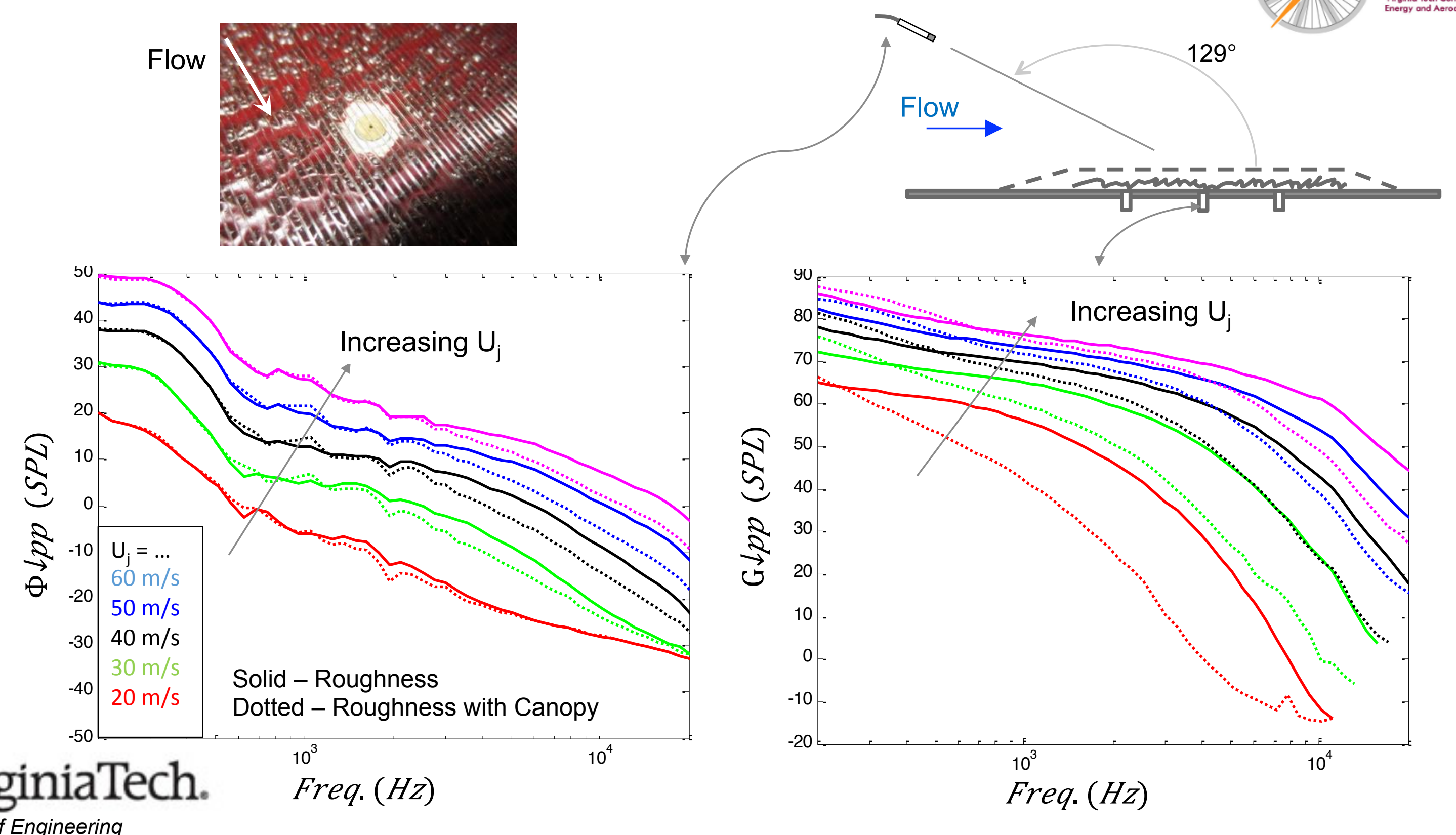




\section{The Idea}

The canopy can greatly suppress surface pressure fluctuations. Would it therefore not also suppress trailing edge noise?

How could a canopy be applied to an airfoil?

Could the hoped-for beneficial effects be achieved without significant adverse effects on the aerodynamics?

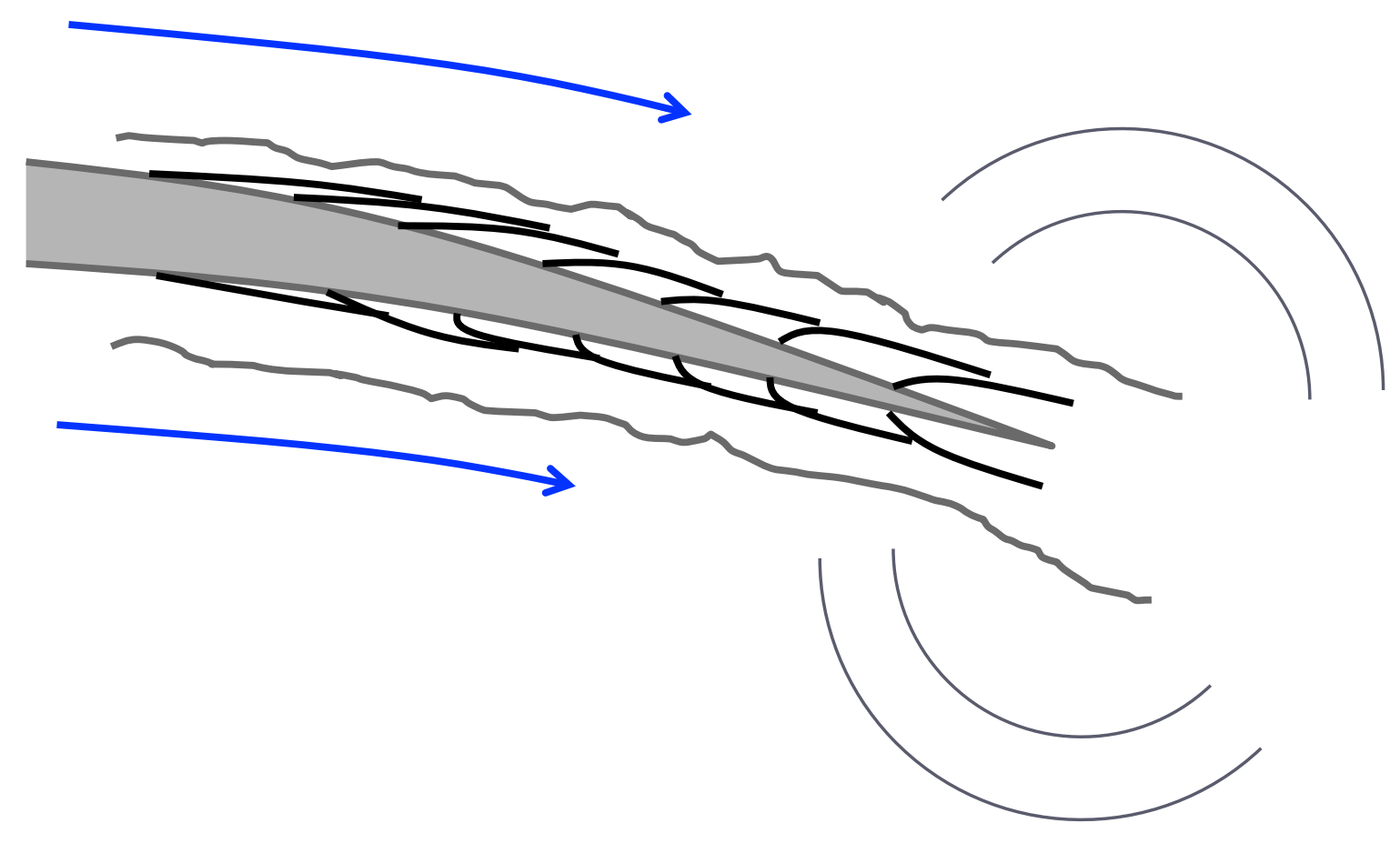




\section{Two Practical Concepts}

Flow-aligned elements on top and bottom surfaces manipulate boundary layers

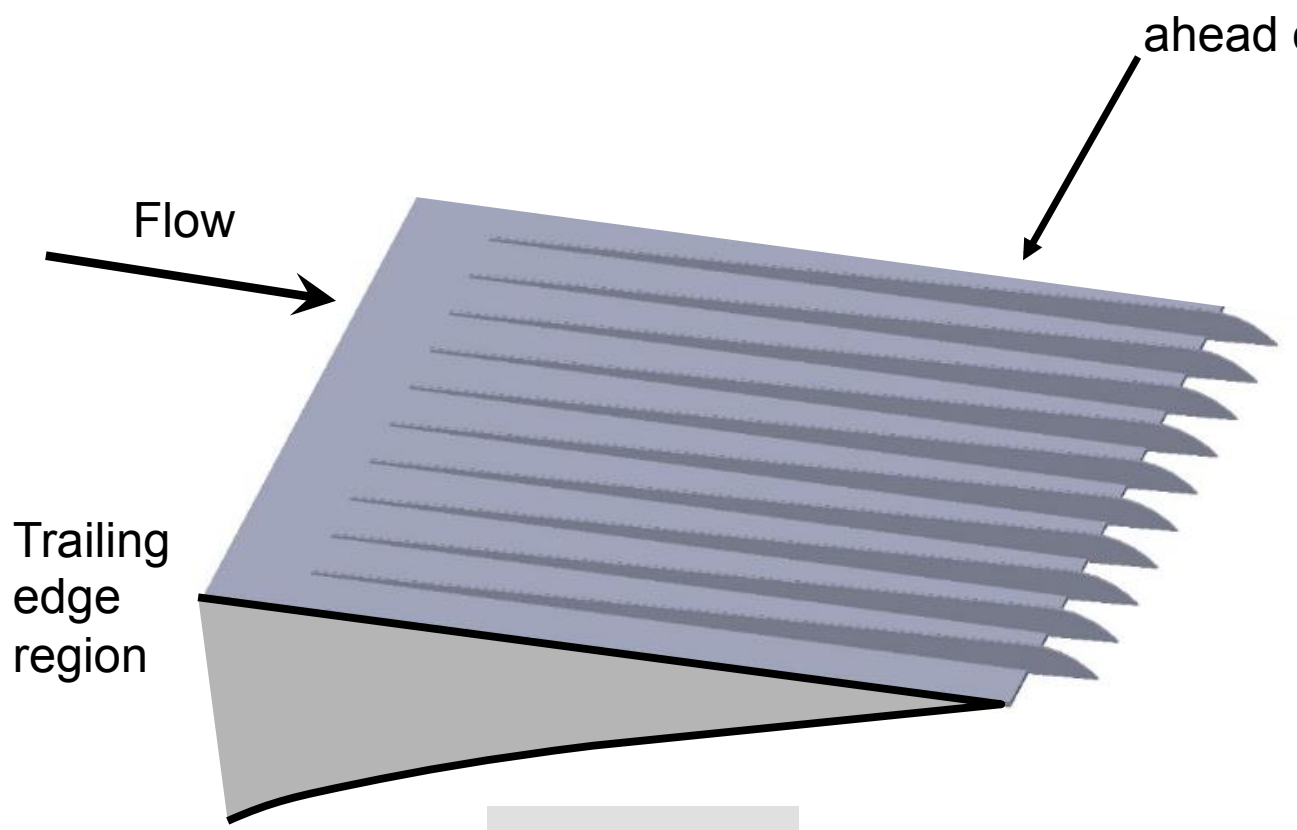

Finlets

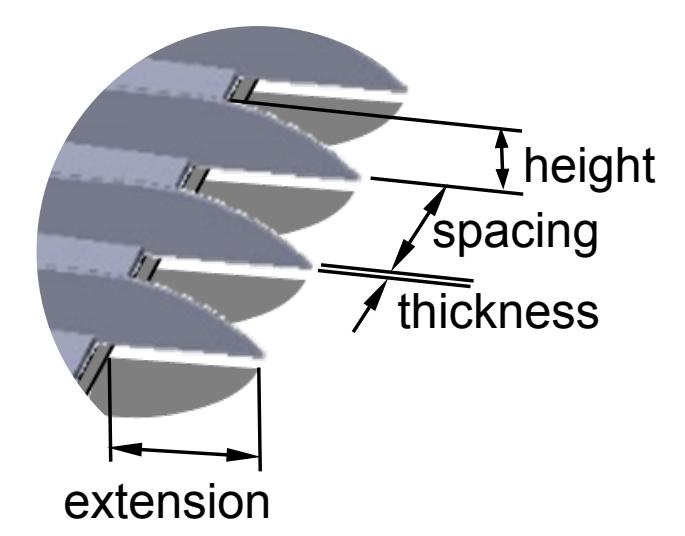

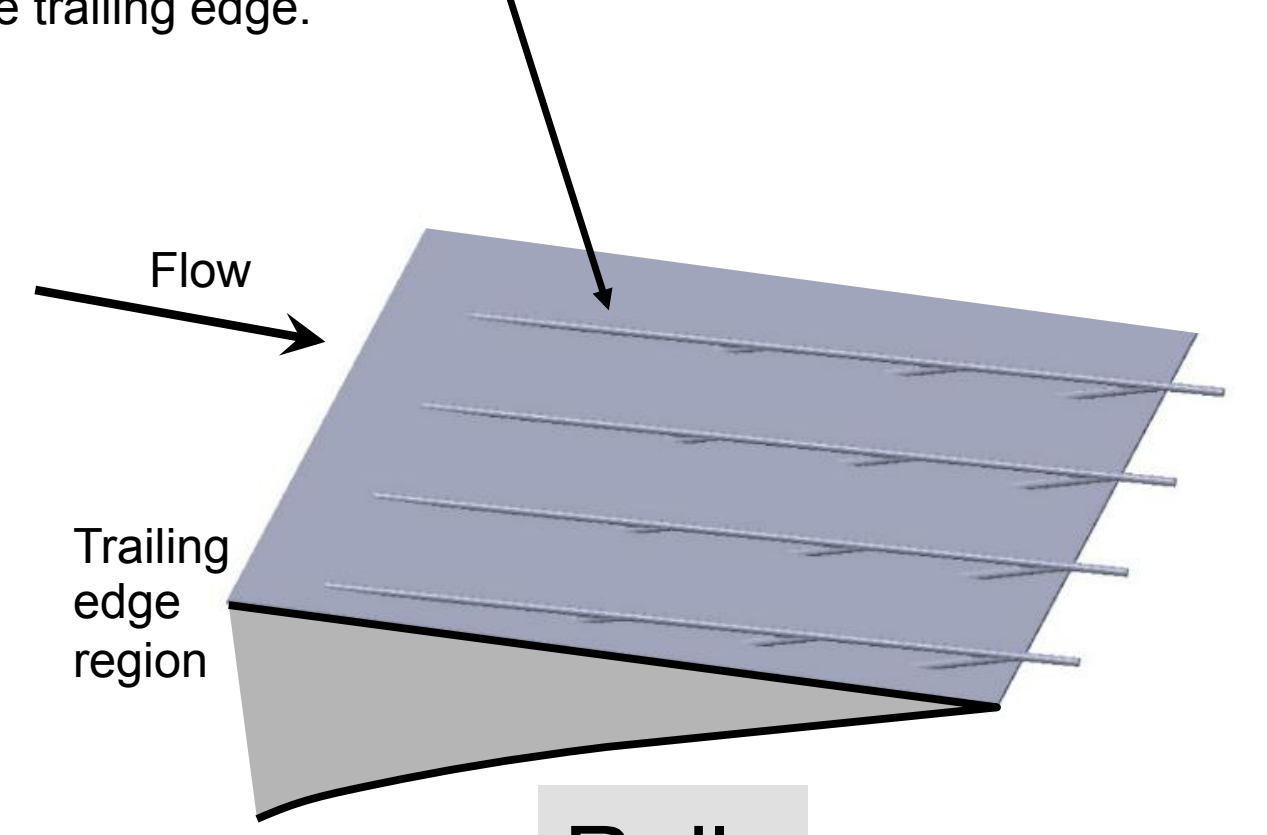

Rails

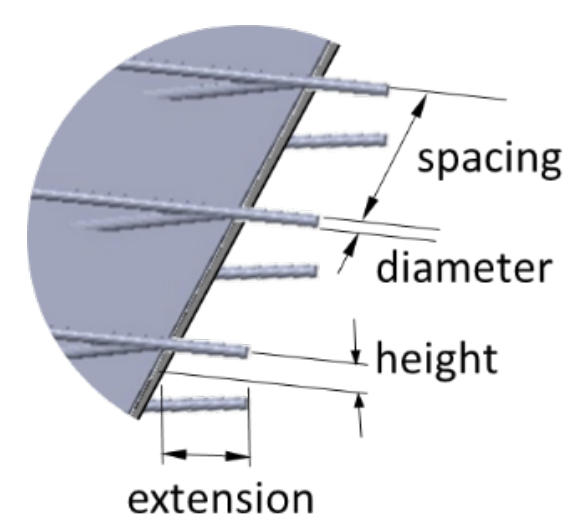




\section{The Experiment}

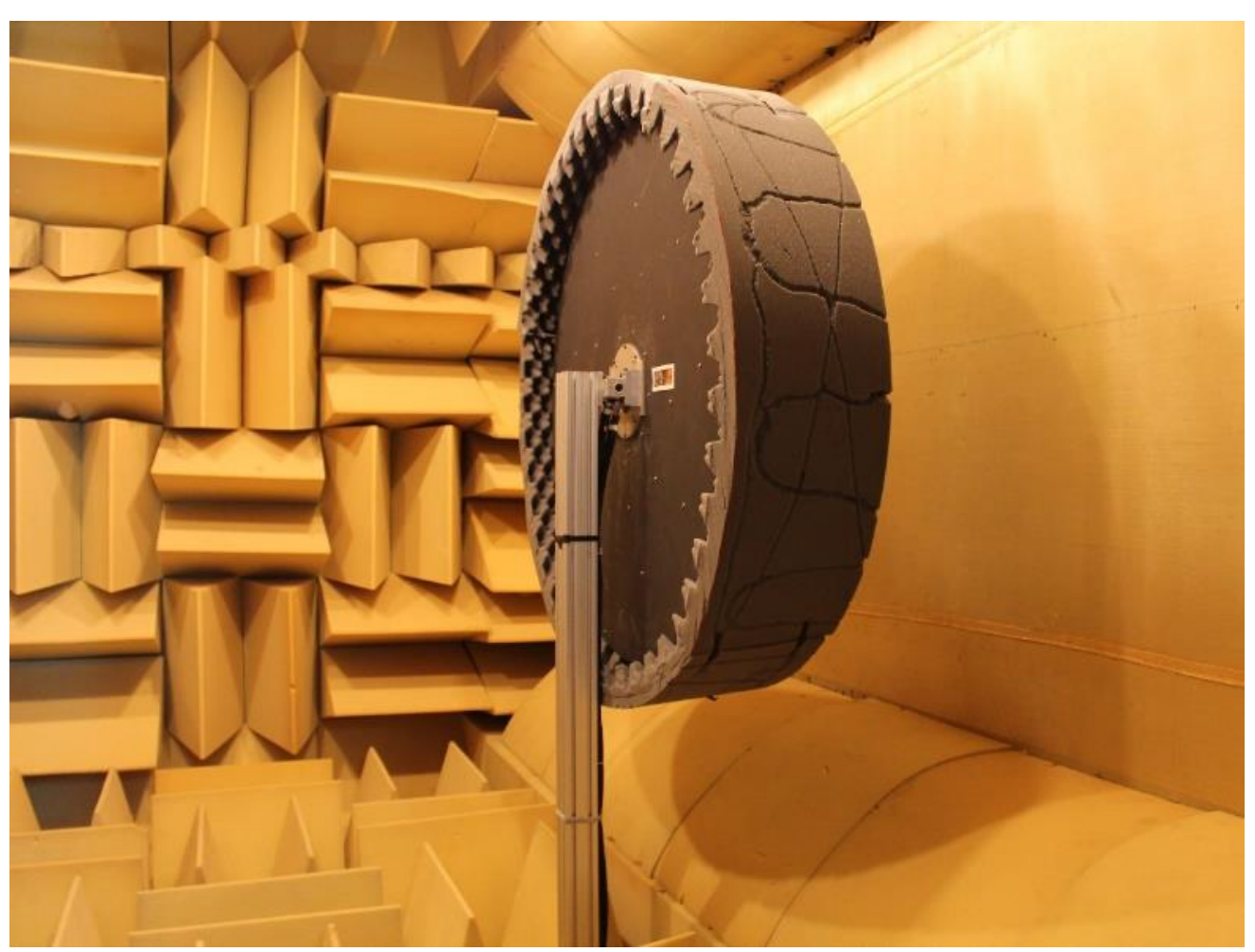

- 117 microphone phase array for far field acoustics

- Surface pressure taps for $C \downarrow p$ and lift

- Wake rake for drag measurements 핑. VirginiaTech.

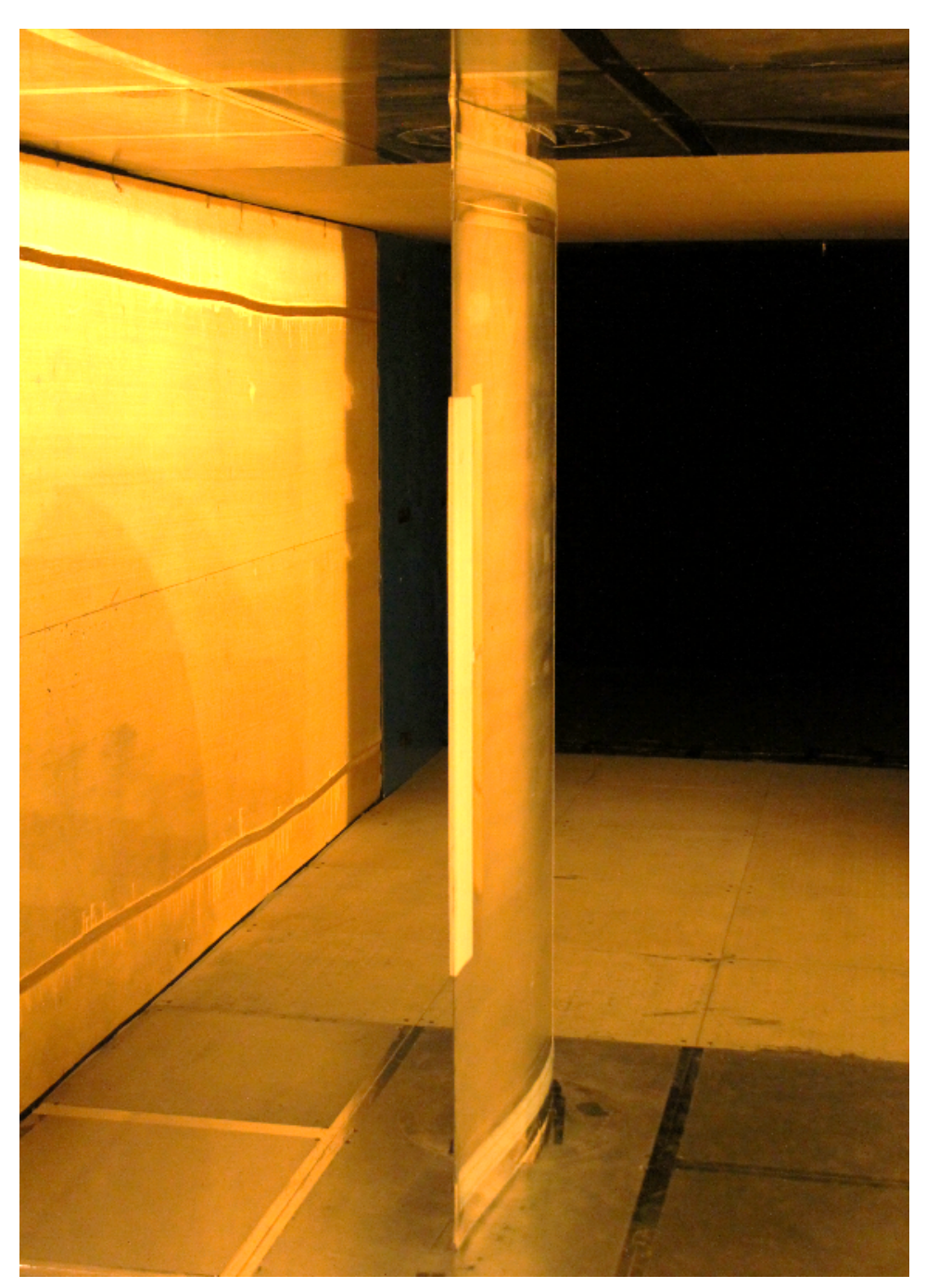

- 0.8-m chord DU96-W180 airfoil

- Tripped $(0.5 \mathrm{~mm}$ zigzag tape) at $5 \% / 10 \%$ chord

- Flow conditions $-M=0.15,0.18(\operatorname{Re} \approx 2.5 \mathrm{M}, 3 \mathrm{M})$

- $\alpha$ from $-4^{\circ}$ to $15^{\circ}, \alpha \downarrow$ zero lift $=-2.5^{\circ}$ 


\section{Clean Airfoil}

Integrated Spectra

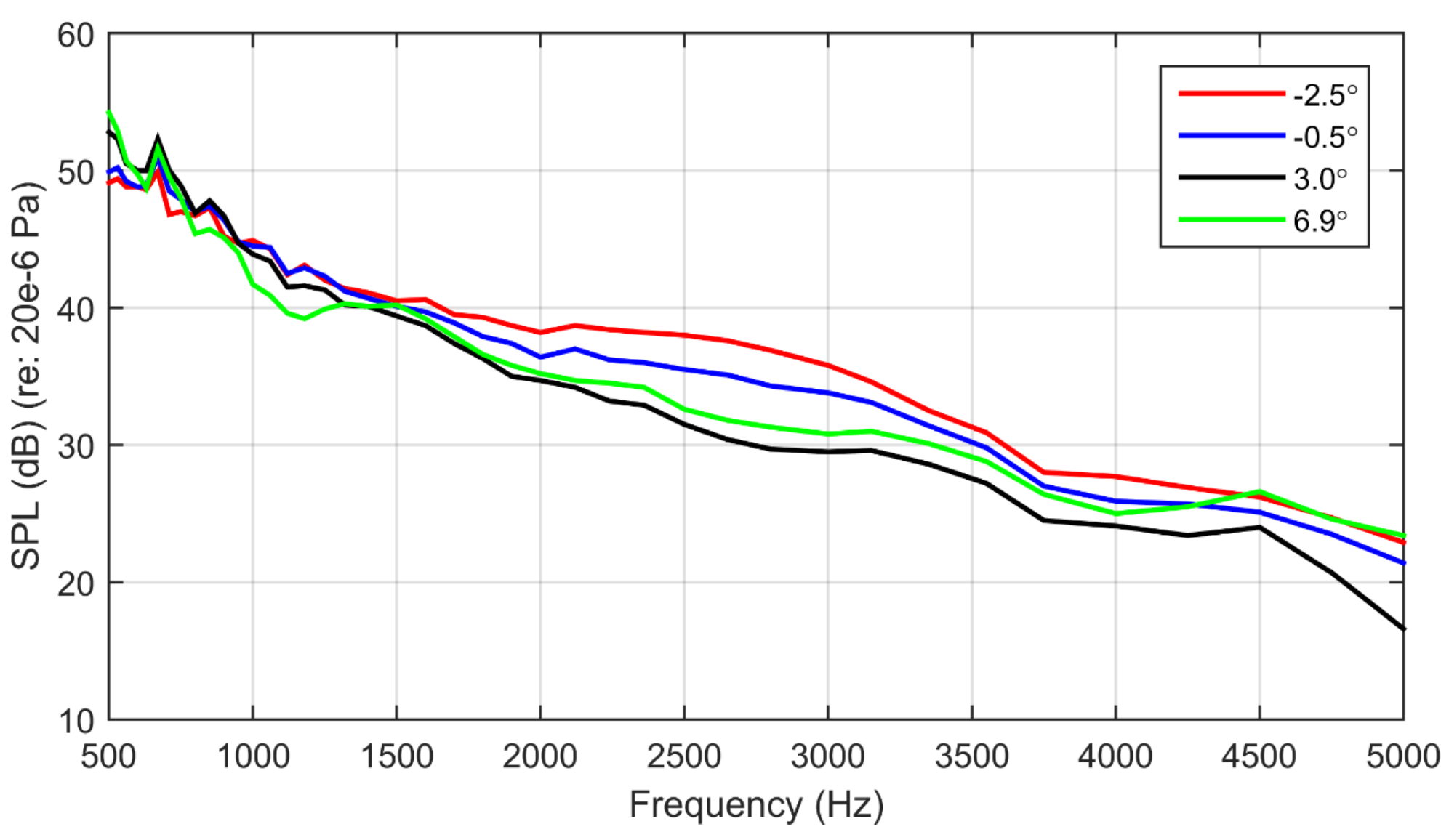

Inj.

\section{$3000 \mathrm{~Hz}$}

$-2.5^{\circ}$
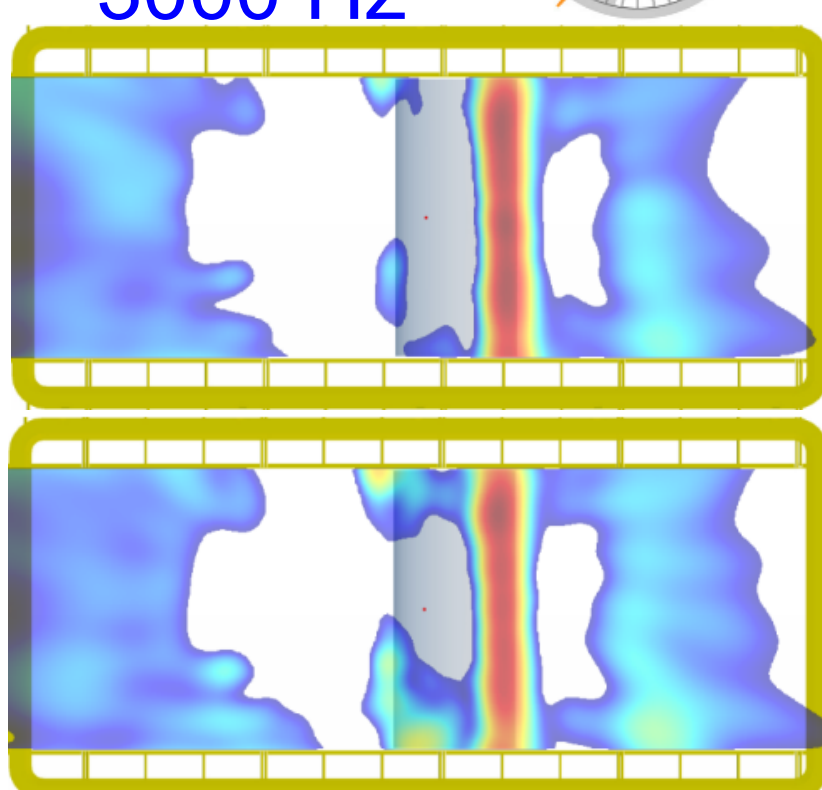

$-0.5^{\circ}$

$3.0^{\circ}$

$6.9^{\circ}$

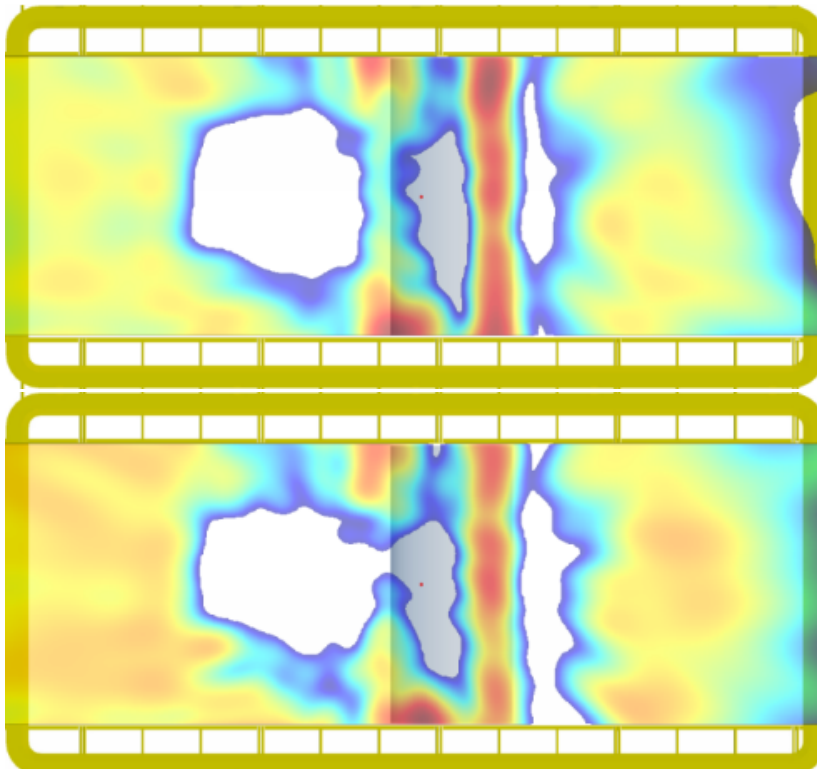




\section{Finlets - Configuration 5}

Thickness $-0.5 \mathrm{~mm}$

Spacing $-4 \mathrm{~mm}$

Height $-4 \mathrm{~mm}$

$10 \mathrm{~mm}$ Extension

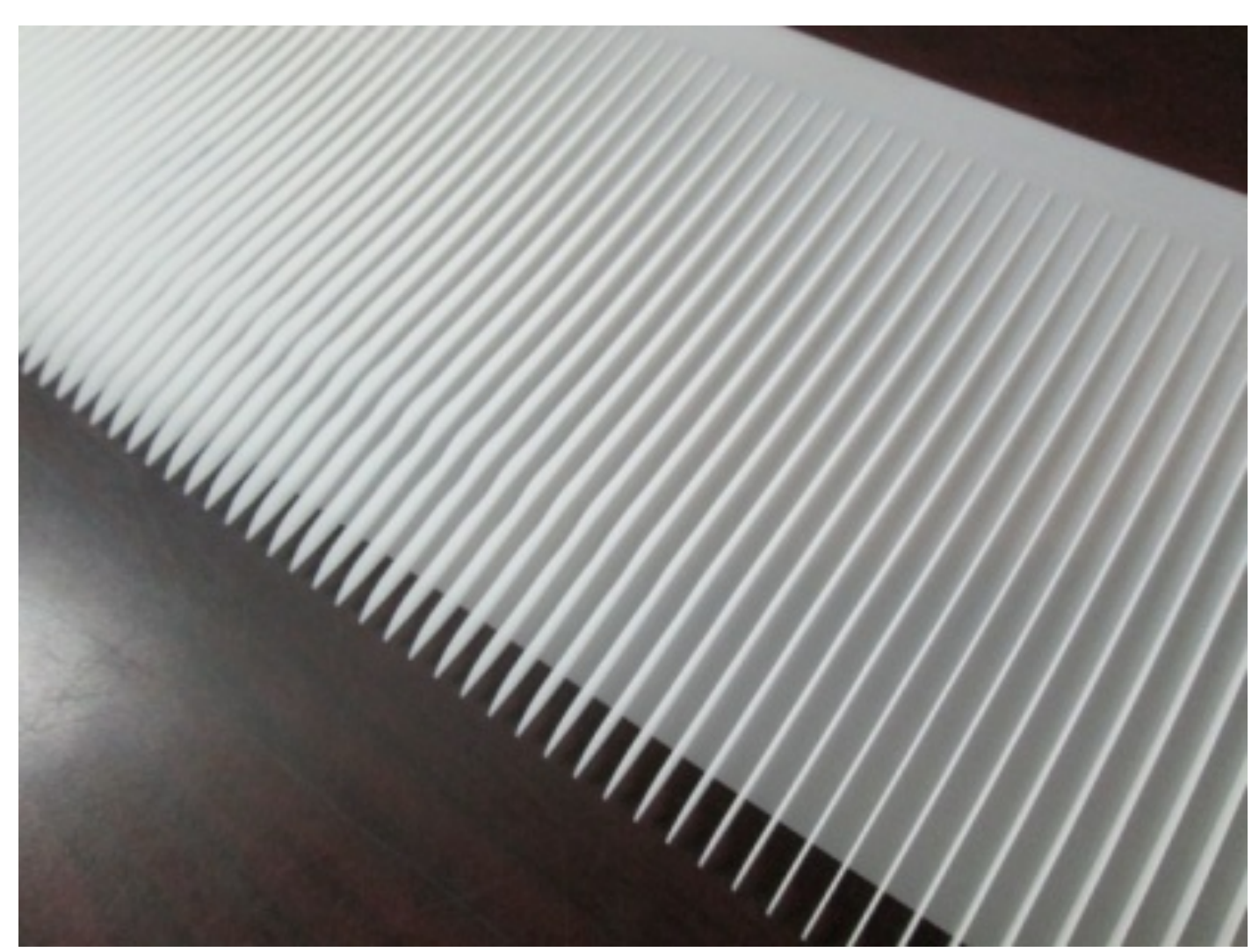

핑 VirginiaTech.

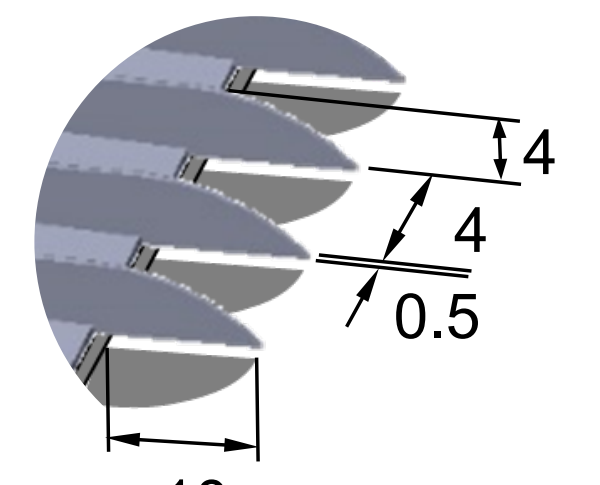

4. CREATe

10

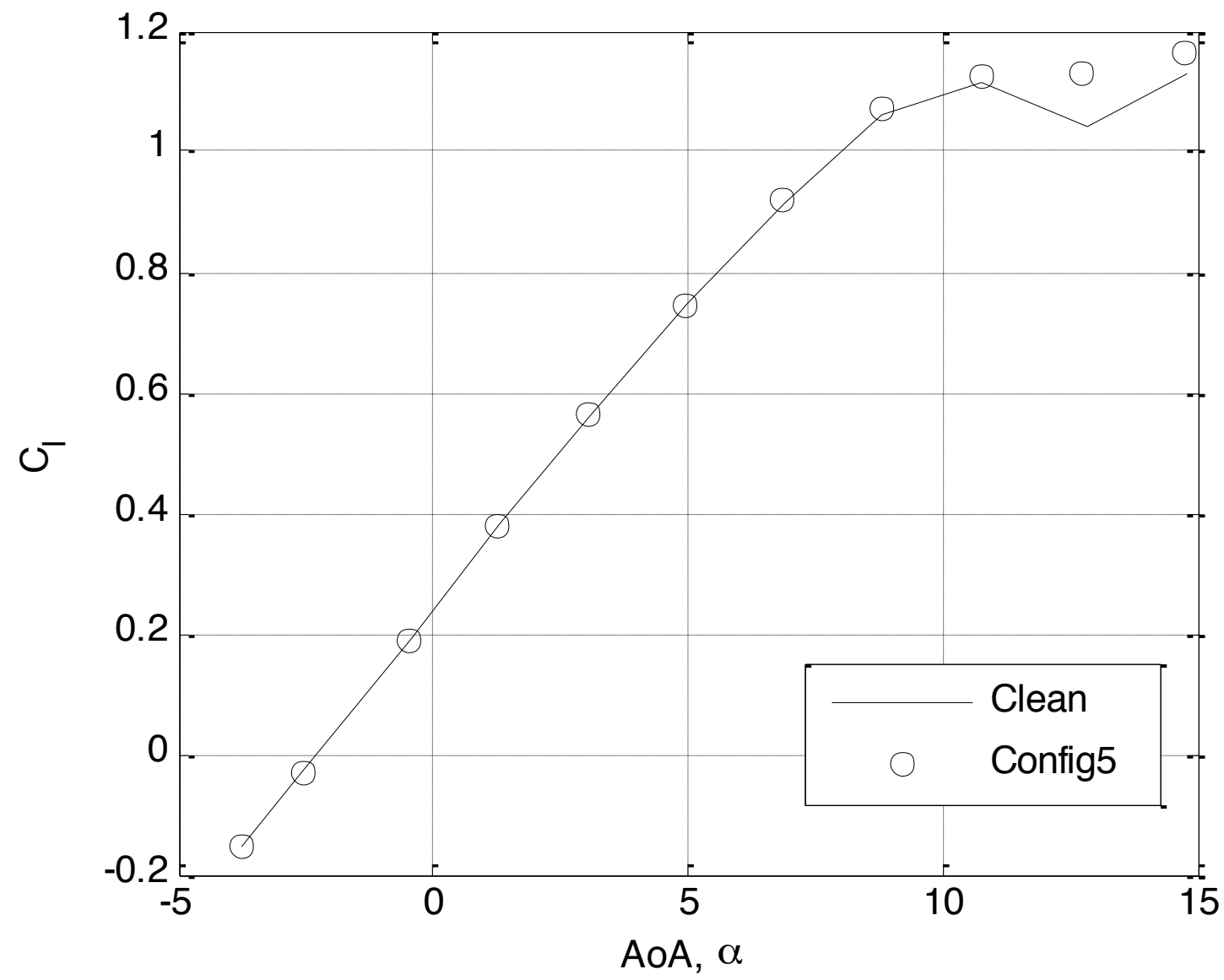




\section{Finlets - Configuration 5}

$$
\operatorname{Re}=3 \mathrm{M}, \text { Tripped, } 3000 \mathrm{~Hz}
$$

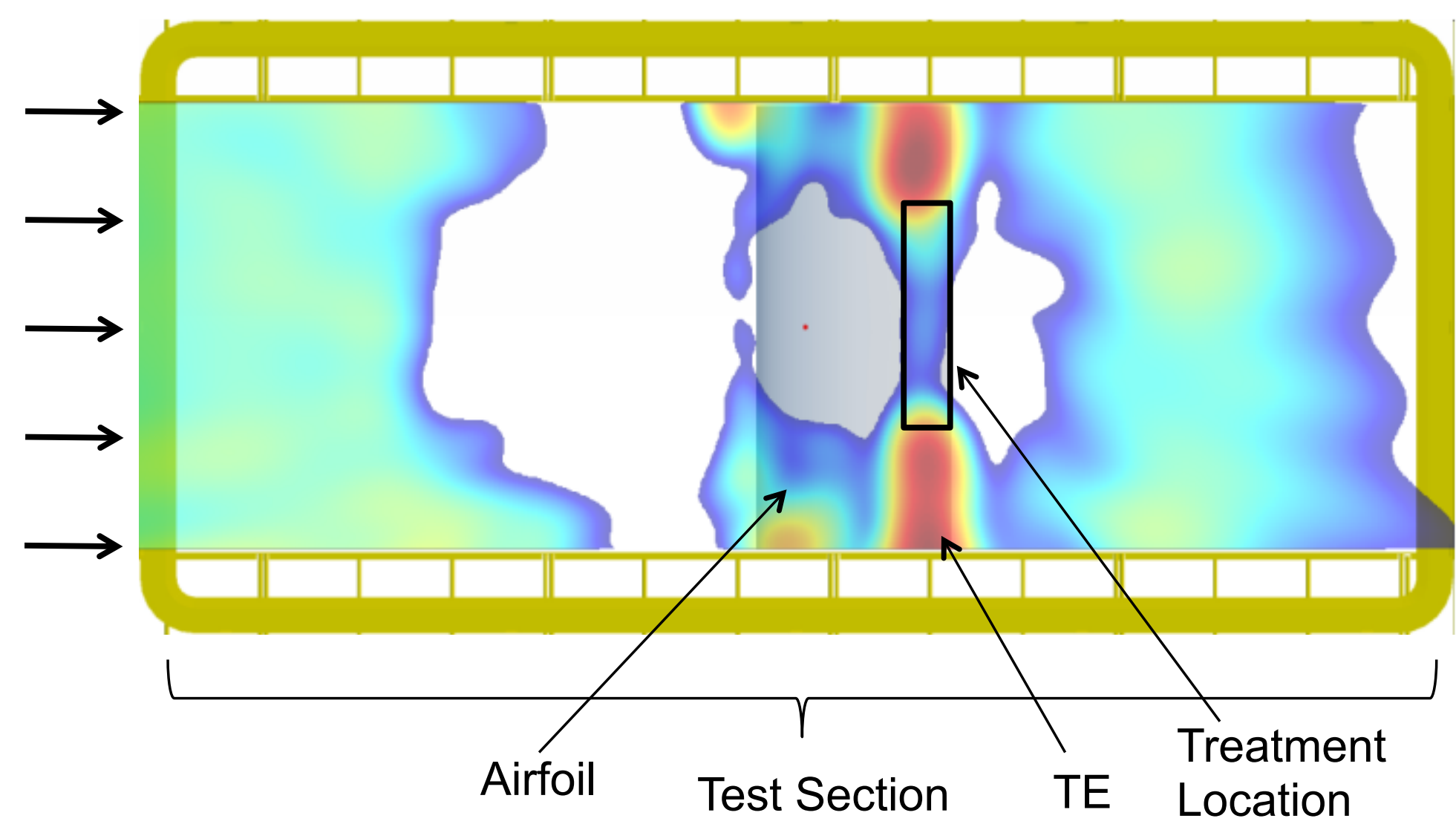

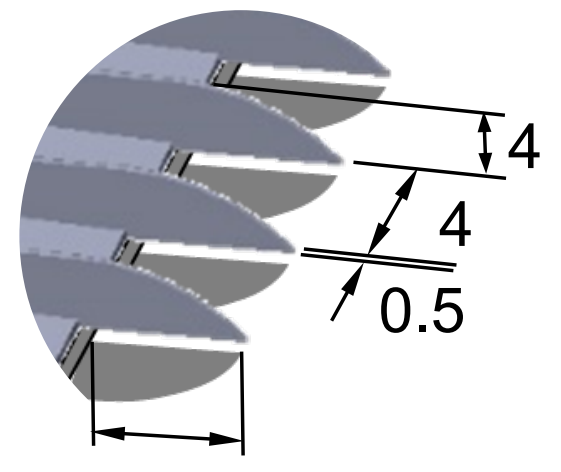

Virginia Tech Center for Renewwoble

10 Thickness $-0.5 \mathrm{~mm}$

SPL [dB]

30
29

Spacing $-4 \mathrm{~mm}$

Height $-4 \mathrm{~mm}$

10mm Extension $(\delta \approx 20 \mathrm{~mm})$

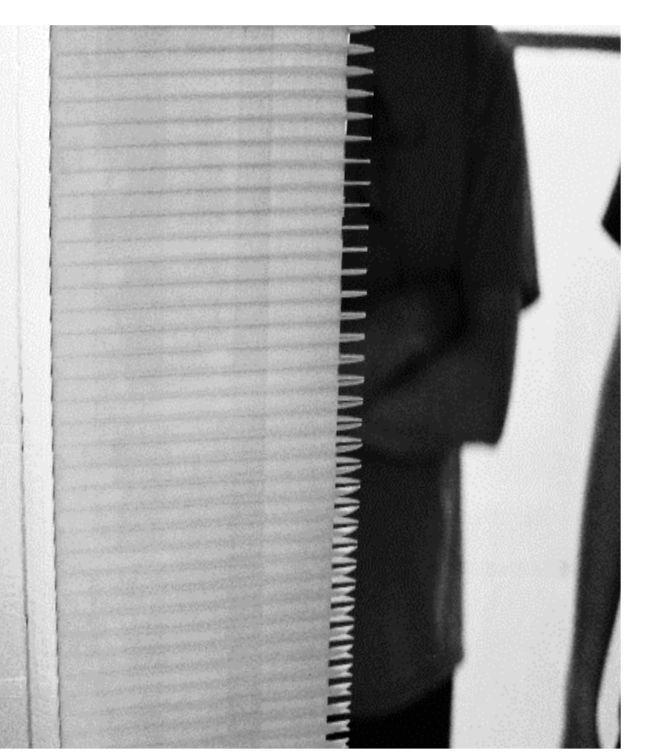




\section{Finlets - Configuration 5}

$$
\operatorname{Re}=3 \mathrm{M}, \text { Tripped, } 3000 \mathrm{~Hz}
$$

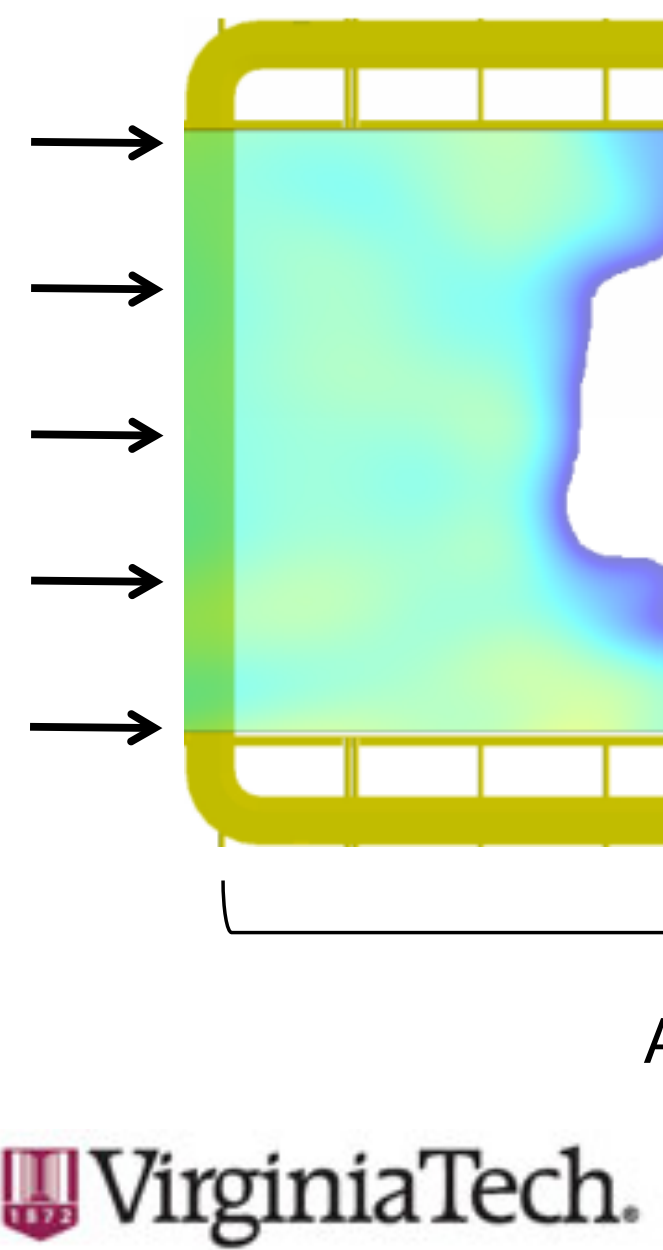

Airfoil

Test Section

$-0.5^{\circ}$
TE

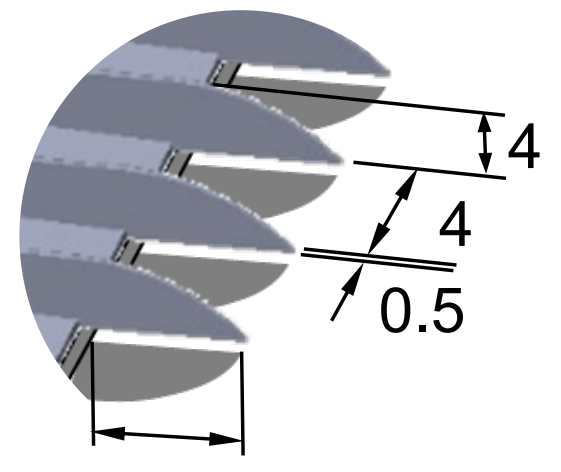

Virgini Tech Center for Renewoble
SPL [dB]

30
29

$-28$

27

$-26$

25

24

23

22

21
20
Thickness $-0.5 \mathrm{~mm}$ Spacing $-4 \mathrm{~mm}$ Height $-4 \mathrm{~mm}$ $10 \mathrm{~mm}$ Extension $(\delta \approx 20 \mathrm{~mm})$
Integration Region

10

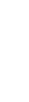




\section{Effect of Configuration 5 Finlet}

Integrated Spectra

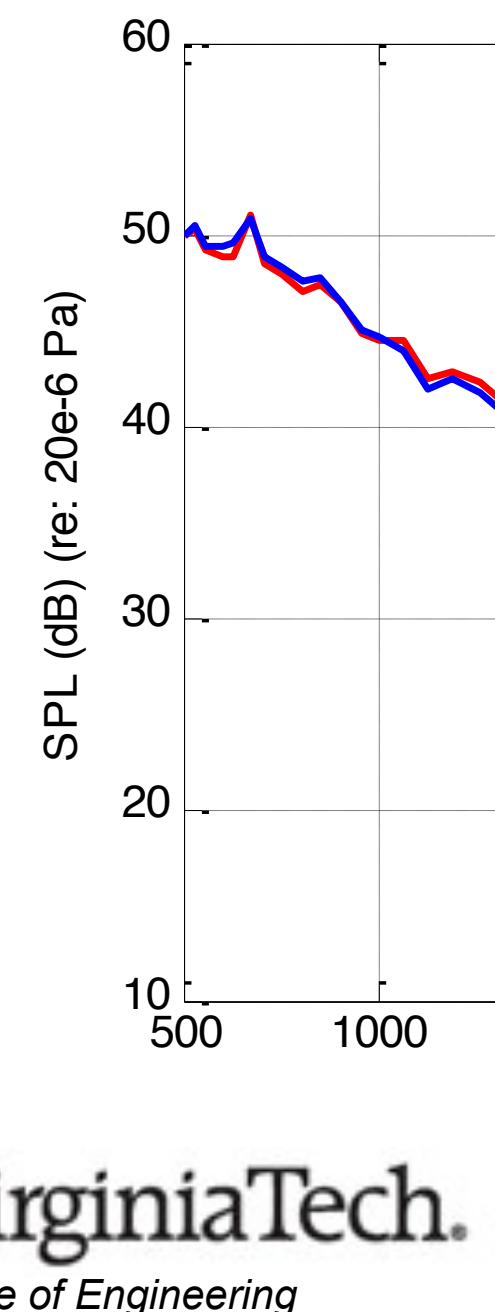

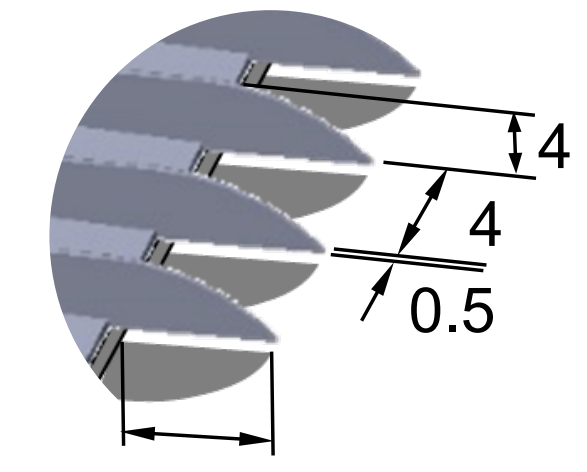

10 Thickness $-0.5 \mathrm{~mm}$ Spacing $-4 \mathrm{~mm}$ Height $-4 \mathrm{~mm}$ $10 \mathrm{~mm}$ Extension $(\delta \approx 15 \mathrm{~mm})$

\section{․ㅣㅇ VirginiaTech.}




\section{Effect of Configuration 5 Finlet}

Integrated Spectra

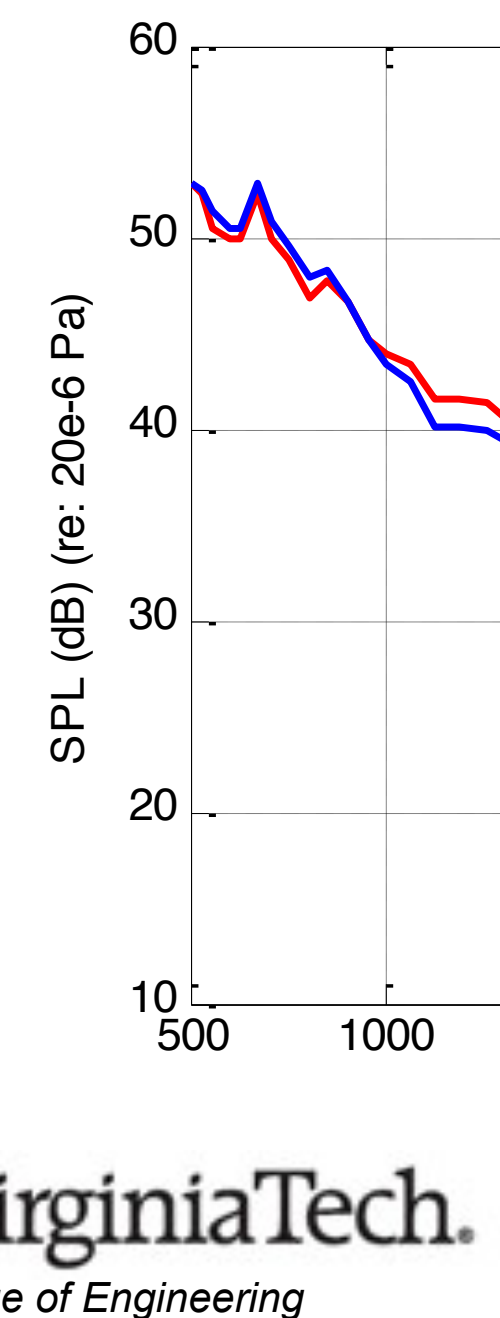

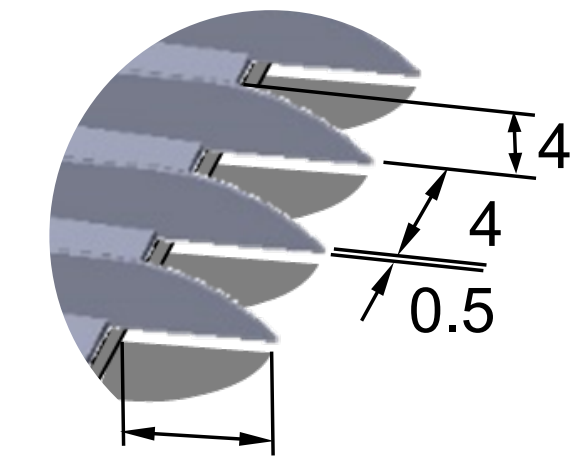

10 Thickness $-0.5 \mathrm{~mm}$ Spacing $-4 \mathrm{~mm}$ Height $-4 \mathrm{~mm}$ $10 \mathrm{~mm}$ Extension $(\delta \approx 30 \mathrm{~mm})$

\section{․ㅣㅇ VirginiaTech.}




\section{Effect of Configuration 5 Finlet}

Integrated Spectra

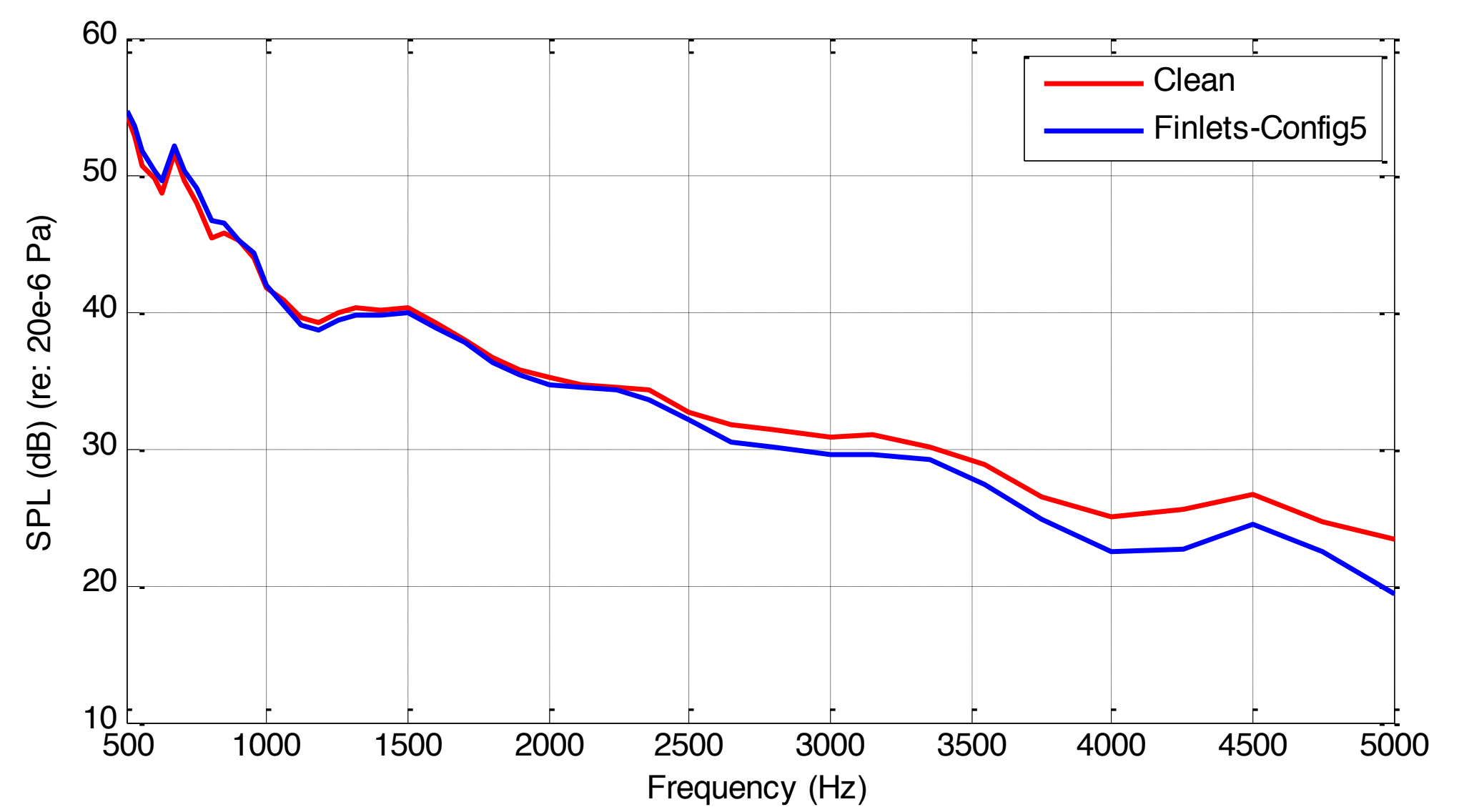

10

Thickness $-0.5 \mathrm{~mm}$ Spacing $-4 \mathrm{~mm}$ Height $-4 \mathrm{~mm}$ $10 \mathrm{~mm}$ Extension $(\delta \approx 40 \mathrm{~mm})$

\section{III)VirginiaTech.}




\section{Effects of Finlet Geometry}

\section{- Spacing}

- In general, smaller finlet spacing improves performance

- However, very small spacings cause vortex shedding

- Height

- Increased height improves performance, particularly at high angle of attack

- Trailling Edge Extension

- Removing the trailing edge extension improves performance, particularly at high angle of attack

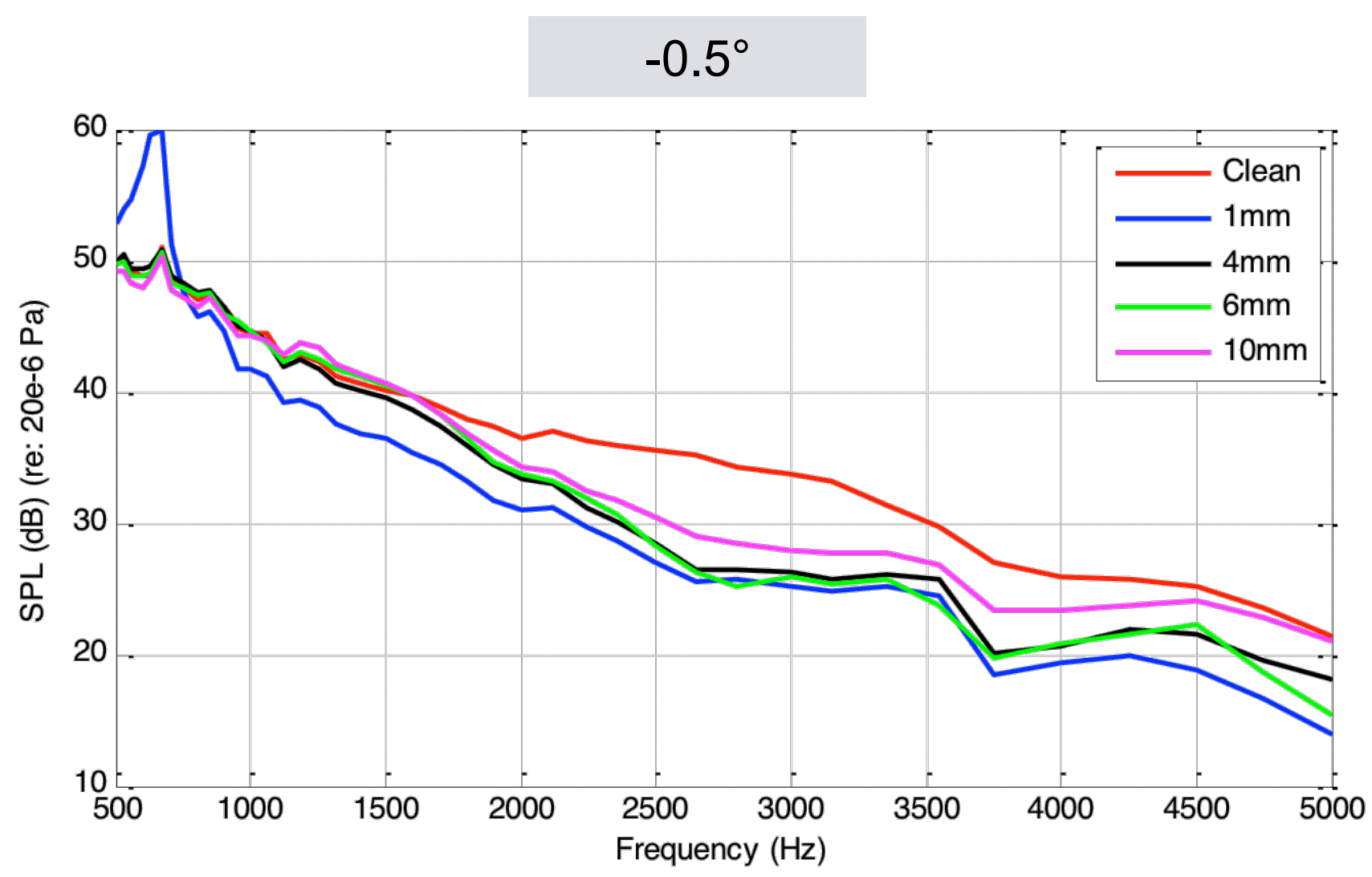




\section{Effects of Finlet Geometry}

- Spacing

- In general, smaller finlet spacing improves performance

- However, very small spacings cause vortex shedding

- Height

- Increased height improves performance, particularly at high angle of attack

- Trailling Edge Extension

- Removing the trailing edge extension improves performance, particularly at high angle of attack

\section{핑 VirginiaTech.}
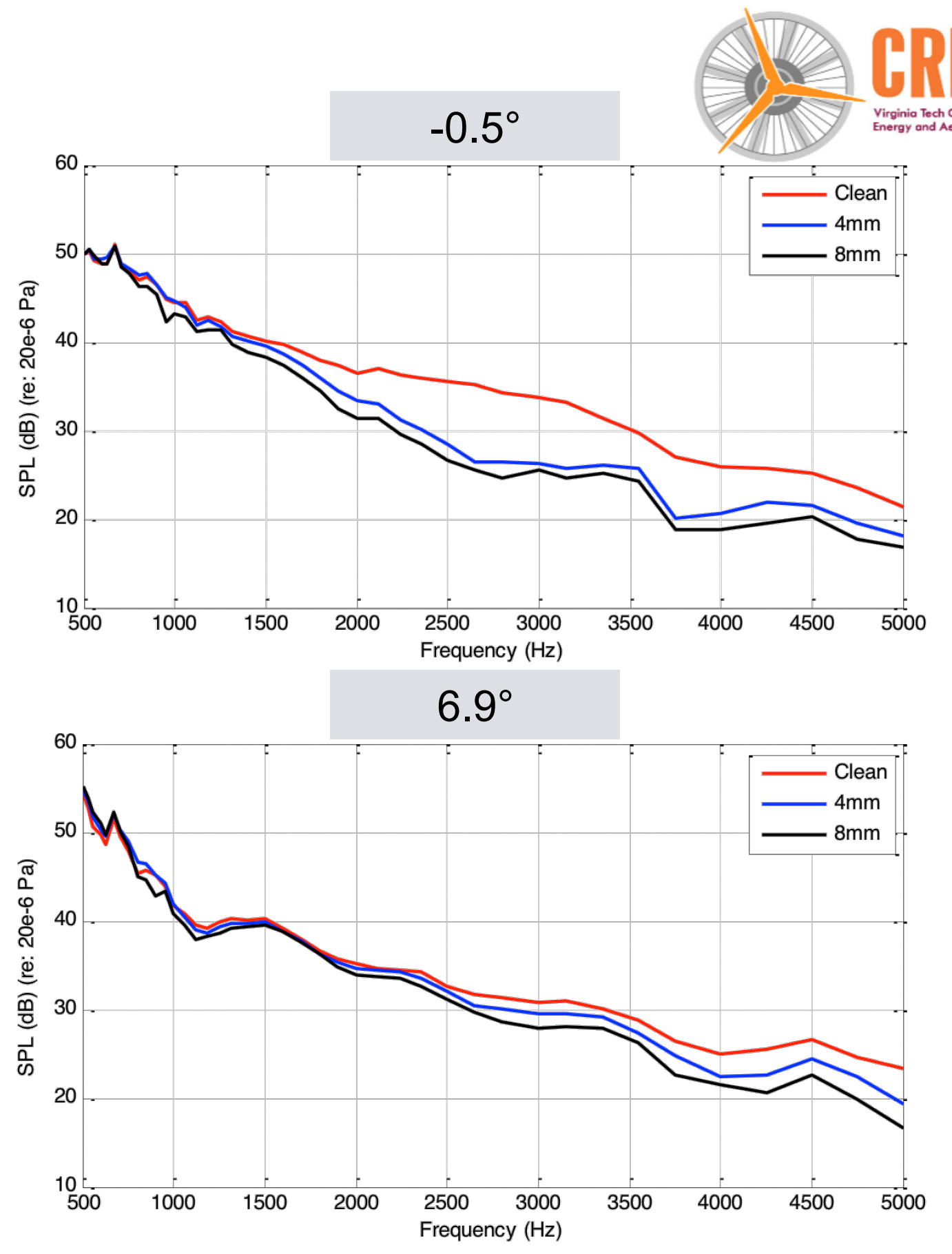


\section{Effects of Finlet Geometry}

- Spacing

- In general, smaller finlet spacing improves performance

- However, very small spacings cause vortex shedding

- Height

- Increased height improves performance, particularly at high angle of attack

\section{- Trailing Edge Extension}

- Removing the trailing edge extension improves performance, particularly at high angle of attack

\section{핑 VirginiaTech.}
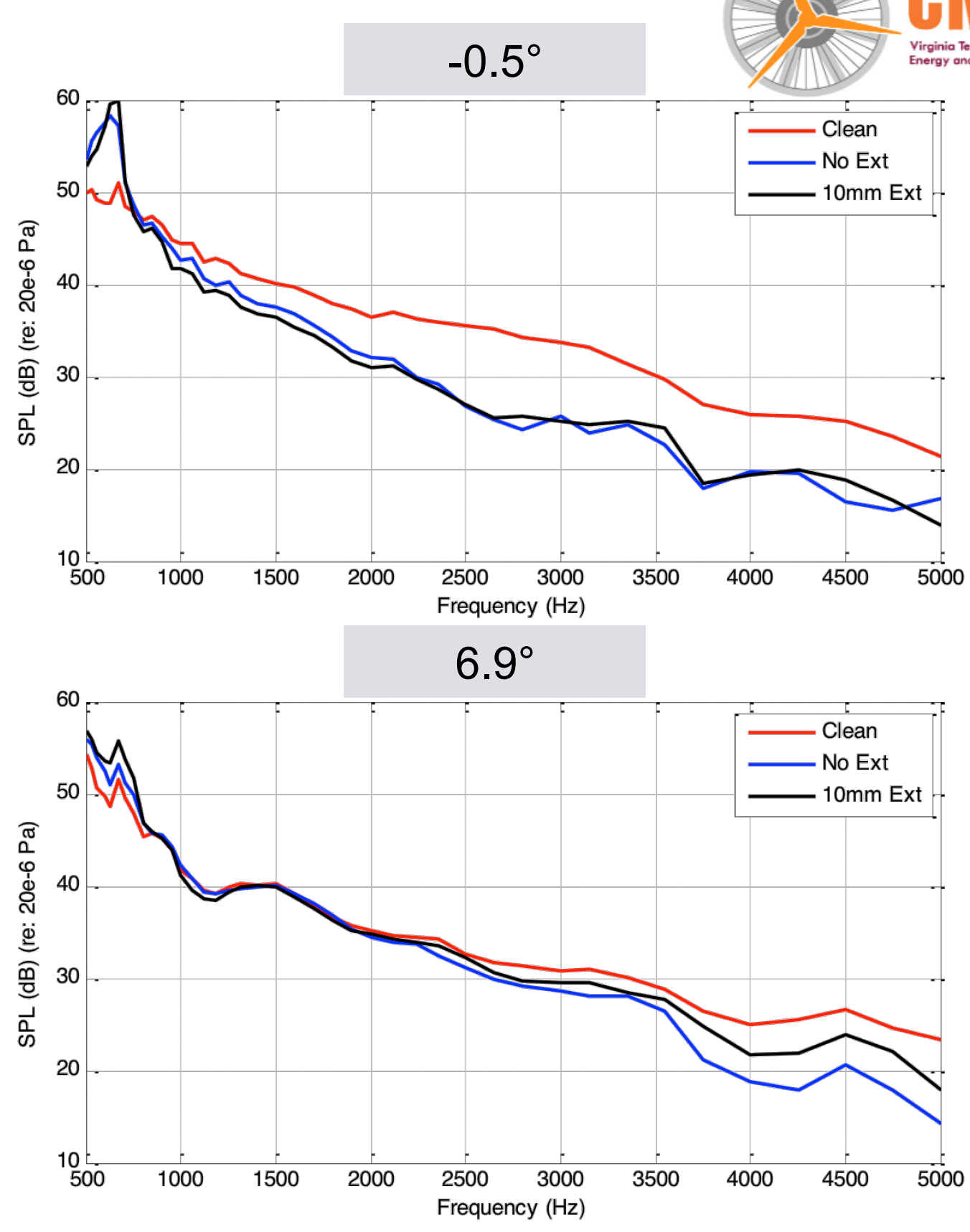


\section{What physical mechanisms are we}

exploiting?

Break up of the boundary layer eddies?

Displacing those structures away from the surface/edge?

Shear sheltering of the edge?

Suppression of trailing edge shedding?

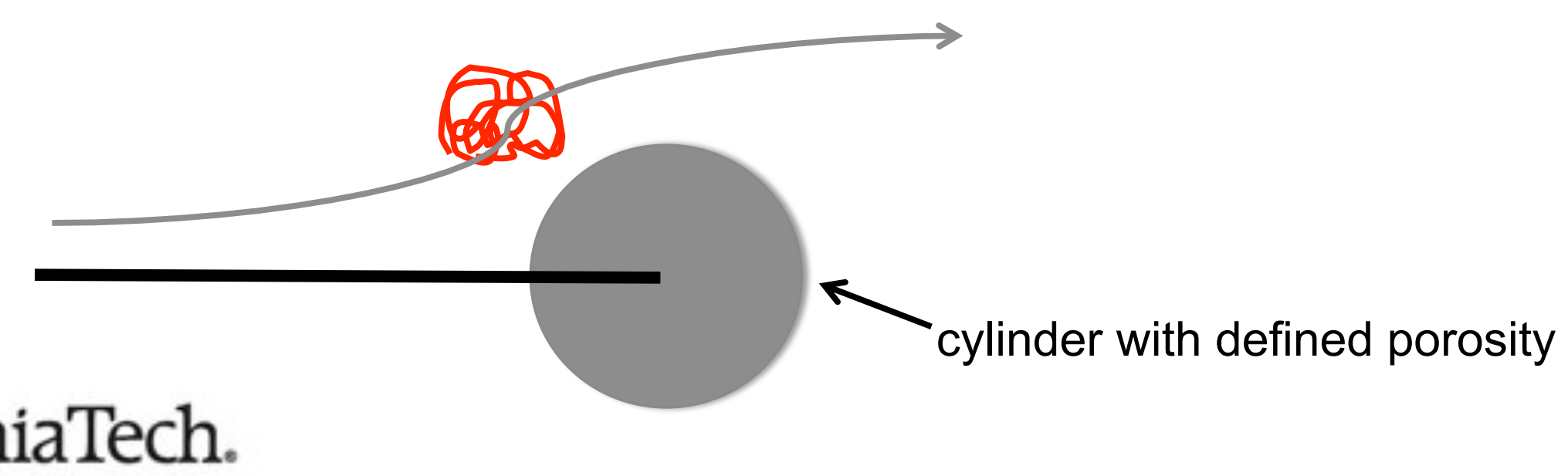




\section{Conclusions}

1. A new, bio-inspired surface treatment for the suppression of trailing edge noise has been demonstrated.

2. The treatment could be combined with existing trailing edge modifications (serrations, etc.) to maximize noise control.

3. The treatment is effective throughout a wide parameter range and is not highly dependent on a particular geometry, but there appears to be strong potential for optimization.

4. The treatment has been shown to be effective over an angle of attack range that extends over 8 degrees from zero lift.

5. Drag data suggests that the impact of finlets is limited to an increase in skin friction from the additional wetted area. 


\section{Extras}




\section{Configurations - Finlets}

\begin{tabular}{|c|c|}
\hline Config\# & Runs \\
\hline 0 & All \\
\hline 2 & 133-154 \\
\hline 10 & 683-704 \\
\hline 3 & $463-484$ \\
\hline 1 & $419-440$ \\
\hline 5 & 243-264 \\
\hline 13 & 804-825 \\
\hline 7 & $331-352$ \\
\hline 11 & $727-748$ \\
\hline 6 & $287-308$ \\
\hline 12 & 760-781 \\
\hline 8 & $375-396$ \\
\hline 9 & $639-660$ \\
\hline 15 & 507-528 \\
\hline $3 S$ & $551-572$ \\
\hline $8 S$ & $980-1001$ \\
\hline & $\begin{array}{l}1112-1122 \\
1134-1144\end{array}$ \\
\hline
\end{tabular}

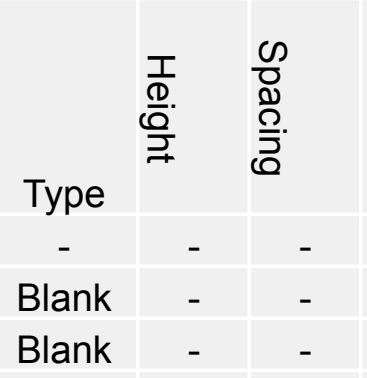

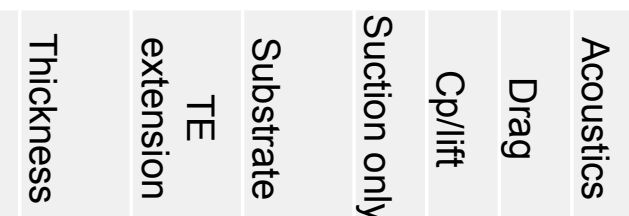

\begin{abstract}
Fin 4
\end{abstract}

Fin 4

Fin

Fin

Fin

Fin

Fin

Fin

Fin

Fin

Fin

Fin

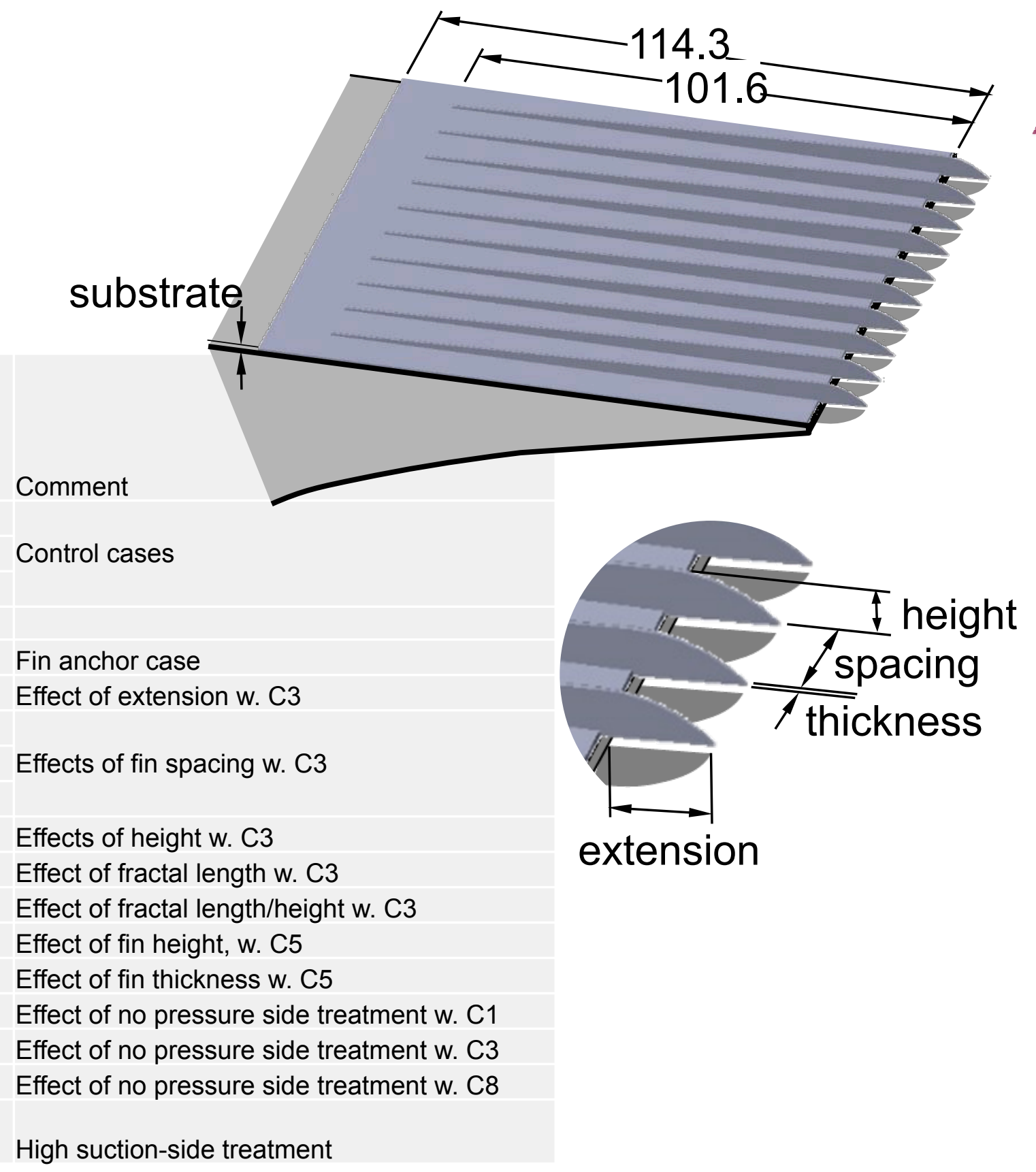




\section{Configurations - Rails}

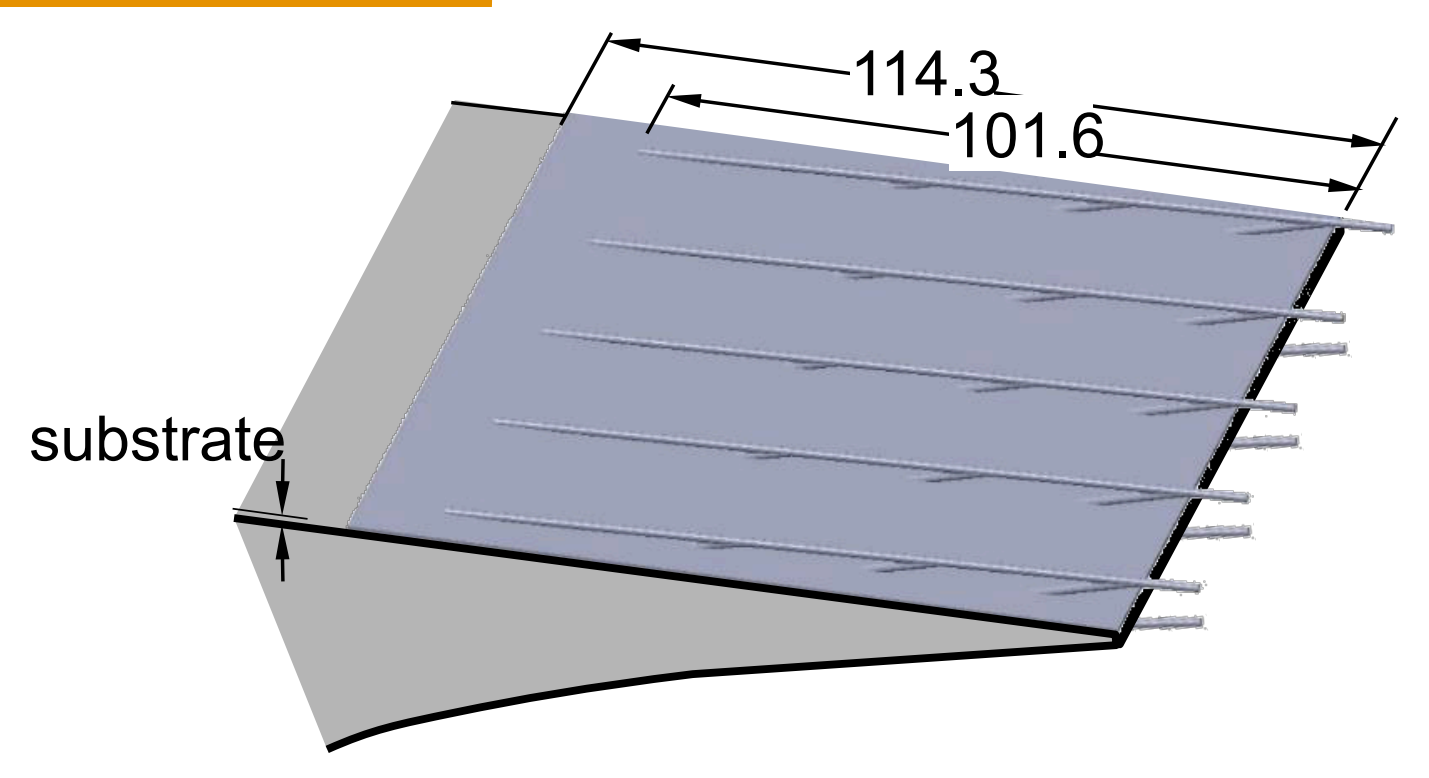

\begin{tabular}{|c|c|c|c|c|c|c|c|c|c|c|c|}
\hline Config\# & Runs & Type & $\frac{\frac{T}{\mathbb{D}}}{\frac{\mathbb{Q}}{D}}$ & 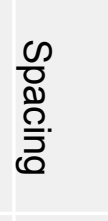 & 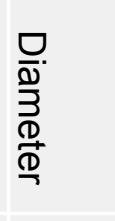 & $\begin{array}{l}\stackrel{\mathbb{D}}{\times} \\
\stackrel{\mathbb{D}}{J} \\
\frac{\mathbb{M}}{\mathrm{O}} \\
\frac{\mathrm{O}}{3}\end{array}$ & $\begin{array}{l}\mathscr{W} \\
\frac{1}{0} \\
\mathscr{W} \\
\stackrel{D}{D} \\
\stackrel{D}{D}\end{array}$ & 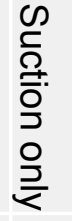 & $\frac{\Omega}{\varrho}$ & ๑̊ & 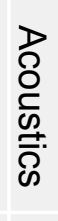 \\
\hline 14 & 848-869 & Rail & 4 & 2.5 & 1.25 & 10 & 0.75 & & $Y$ & - & $Y$ \\
\hline 15 & $936-957$ & Rail & 4 & 2.5 & 1.25 & 0 & 0.75 & - & $Y$ & - & Y \\
\hline 17 & $1024-1045$ & Rail & 8 & 2.5 & 1.25 & 10 & 0.75 & - & $Y$ & - & $Y$ \\
\hline 18 & $1068-1089$ & Rail & 4 & 5 & 2.5 & 10 & 0.75 & - & $Y$ & - & $Y$ \\
\hline 20 & $1211-1232$ & Rail & 8 & 10 & 1.25 & 10 & 0.75 & - & $Y$ & - & Y \\
\hline 19 & $1167-1188$ & Rail & 4 & 2.5 & 1.25 & 10 & 0.75 & - & $Y$ & - & $Y$ \\
\hline Velvet & $892-913$ & Velvet & $\mathrm{n} / \mathrm{a}$ & $\mathrm{n} / \mathrm{a}$ & $\mathrm{n} / \mathrm{a}$ & $\mathrm{n} / \mathrm{a}$ & $\mathrm{n} / \mathrm{a}$ & - & $Y$ & - & $Y$ \\
\hline Velcro & $23-44$ & Velcro & $\mathrm{n} / \mathrm{a}$ & $\mathrm{n} / \mathrm{a}$ & $\mathrm{n} / \mathrm{a}$ & $\mathrm{n} / \mathrm{a}$ & $\mathrm{n} / \mathrm{a}$ & - & $Y$ & - & Y \\
\hline
\end{tabular}

Comment

Rod anchor case

Effect of extension w. C14

Effect of height w. C14

Effect of dia. \& spacing w. C14

Effect of spacing w. C17

Effect of fractal length w. C14

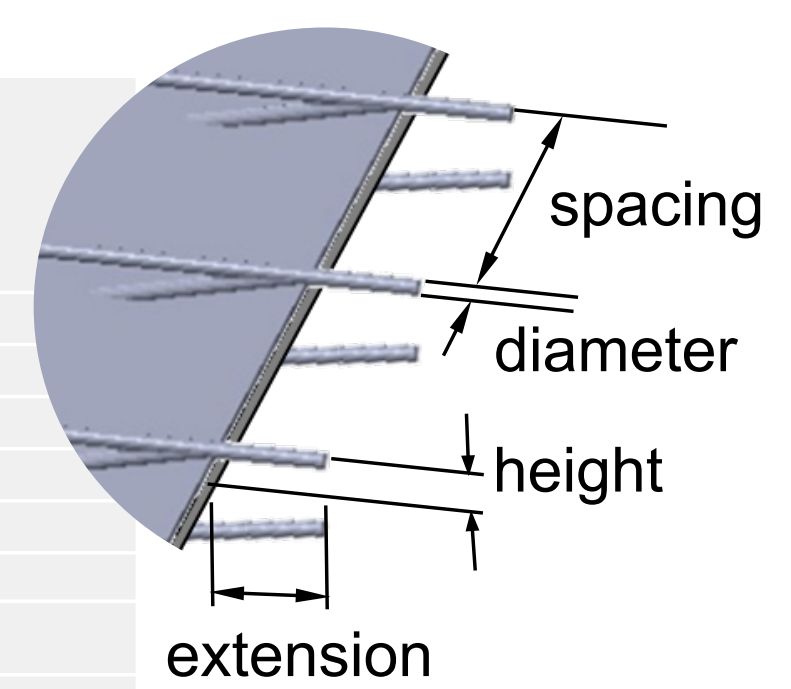




\section{BL Thickness}
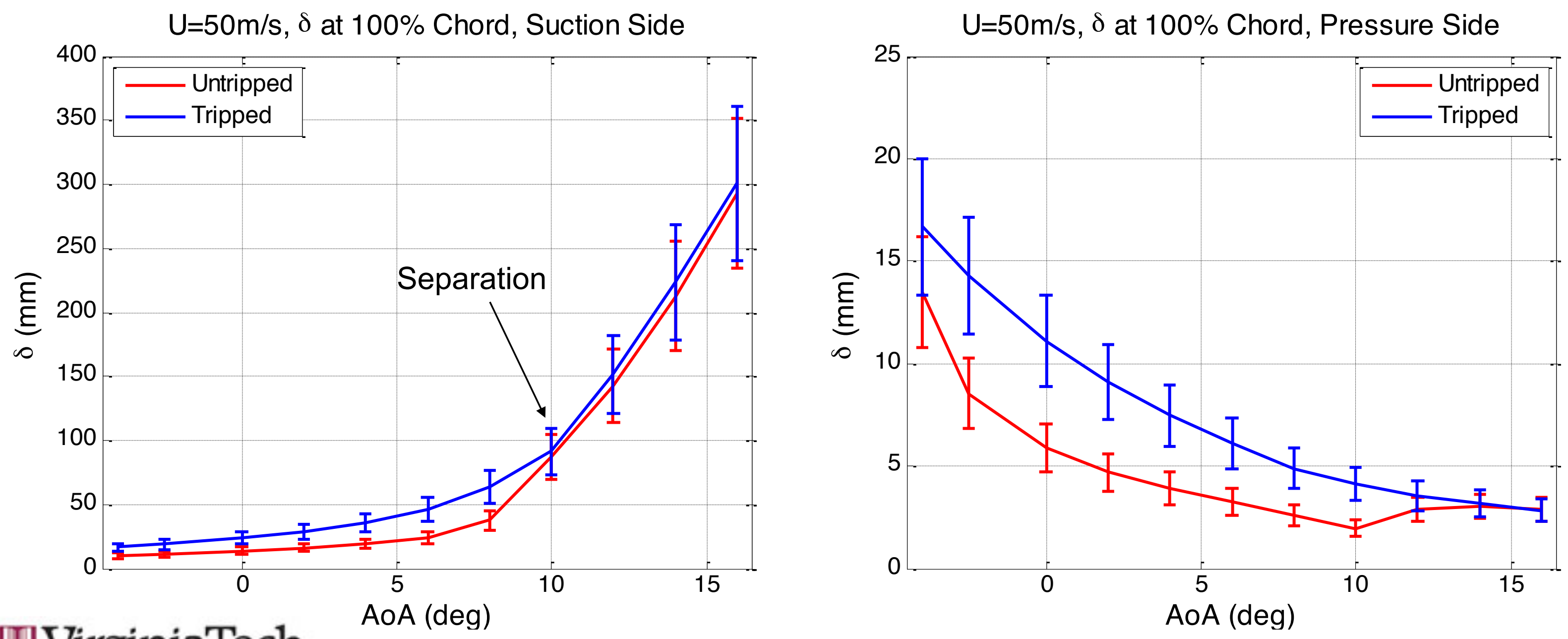


\section{Effect of Configuration 5 Finlet}

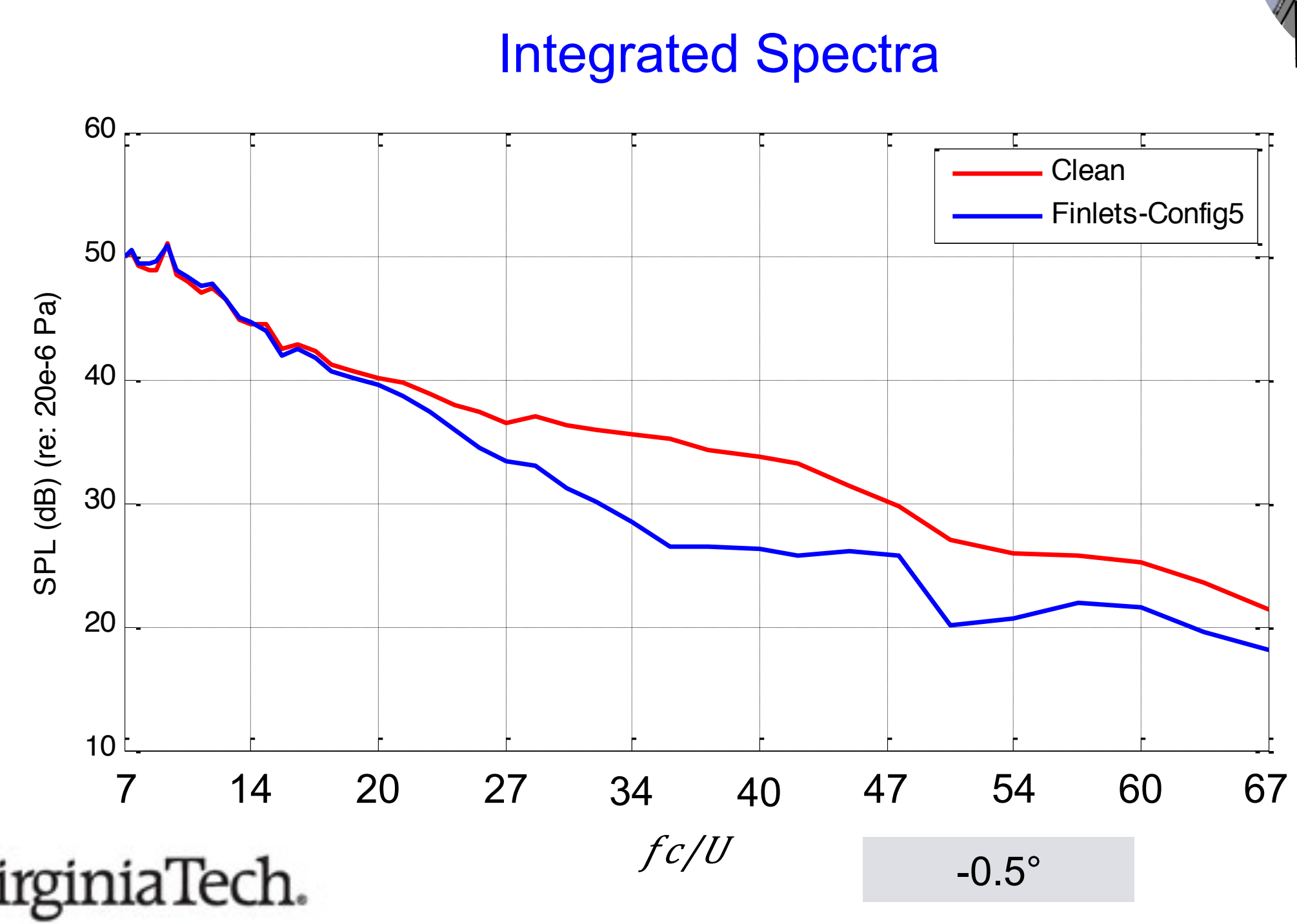

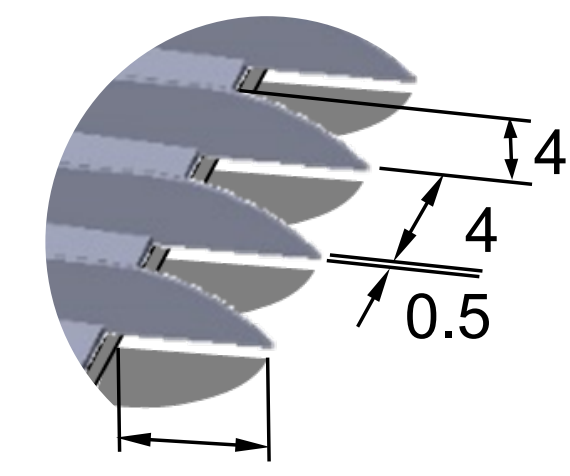

10 Thickness $-0.5 \mathrm{~mm}$ Spacing $-4 \mathrm{~mm}$ Height $-4 \mathrm{~mm}$ $10 \mathrm{~mm}$ Extension $(\delta \approx 15 \mathrm{~mm})$

\section{핑 VirginiaTech.}




\section{Finlets - Effect of Spacing}

Integrated Spectra

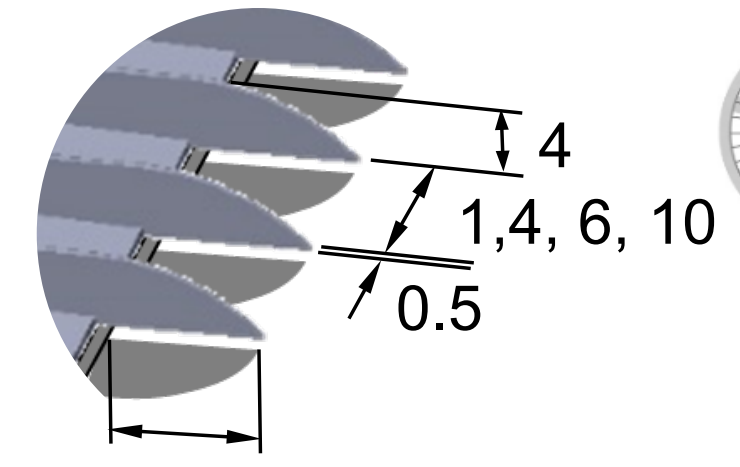

10

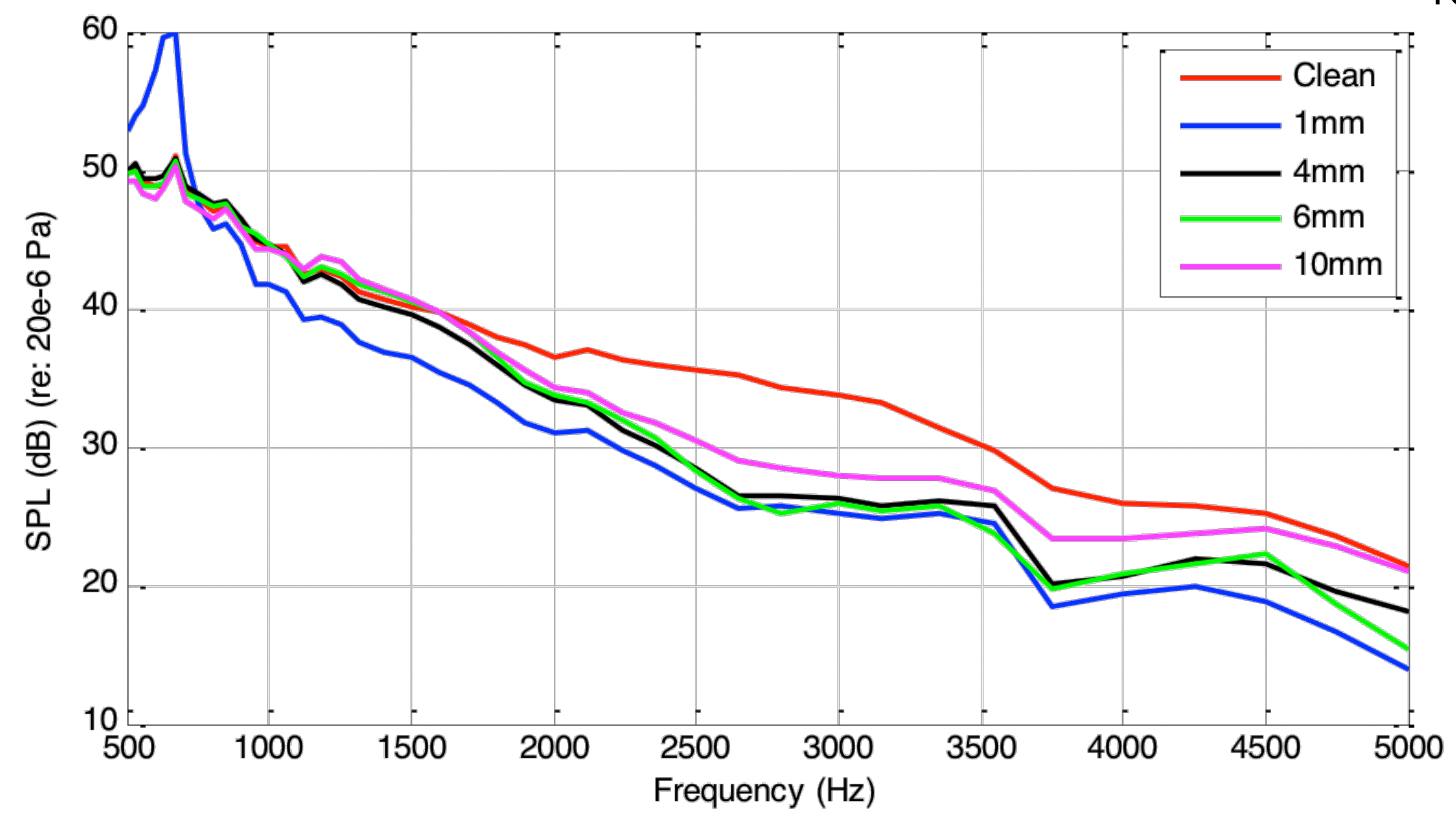

Thickness $-0.5 \mathrm{~mm}$

Spacing $-1,4,6,10 \mathrm{~mm}$

Height $-4 \mathrm{~mm}$

$10 \mathrm{~mm}$ Extension 


\section{Finlets - Effect of Height}

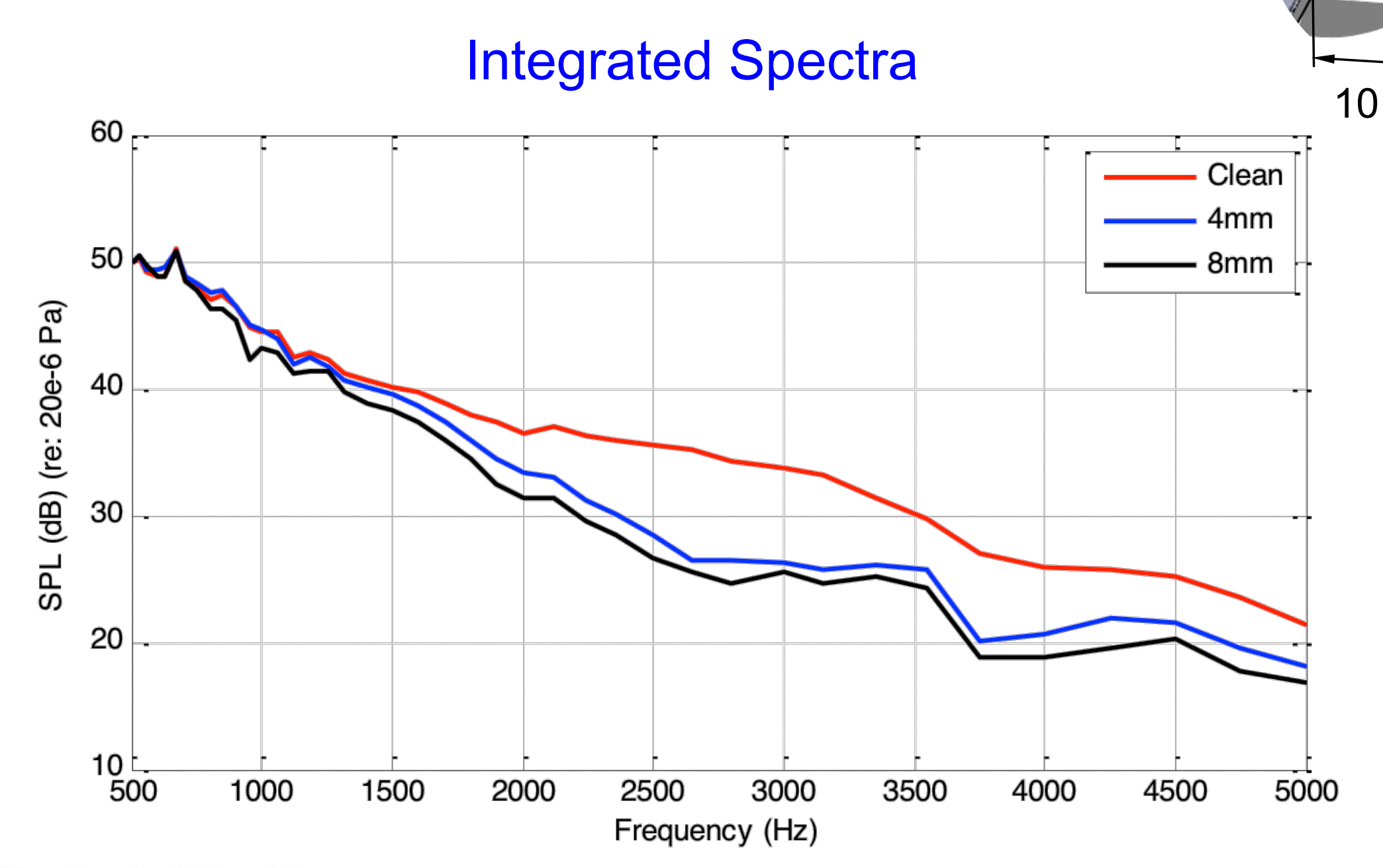

Thickness $-0.5 \mathrm{~mm}$

Spacing $-4 \mathrm{~mm}$

Height $-4,8 \mathrm{~mm}$

$10 \mathrm{~mm}$ Extension 


\section{Finlets - Effect of Height}

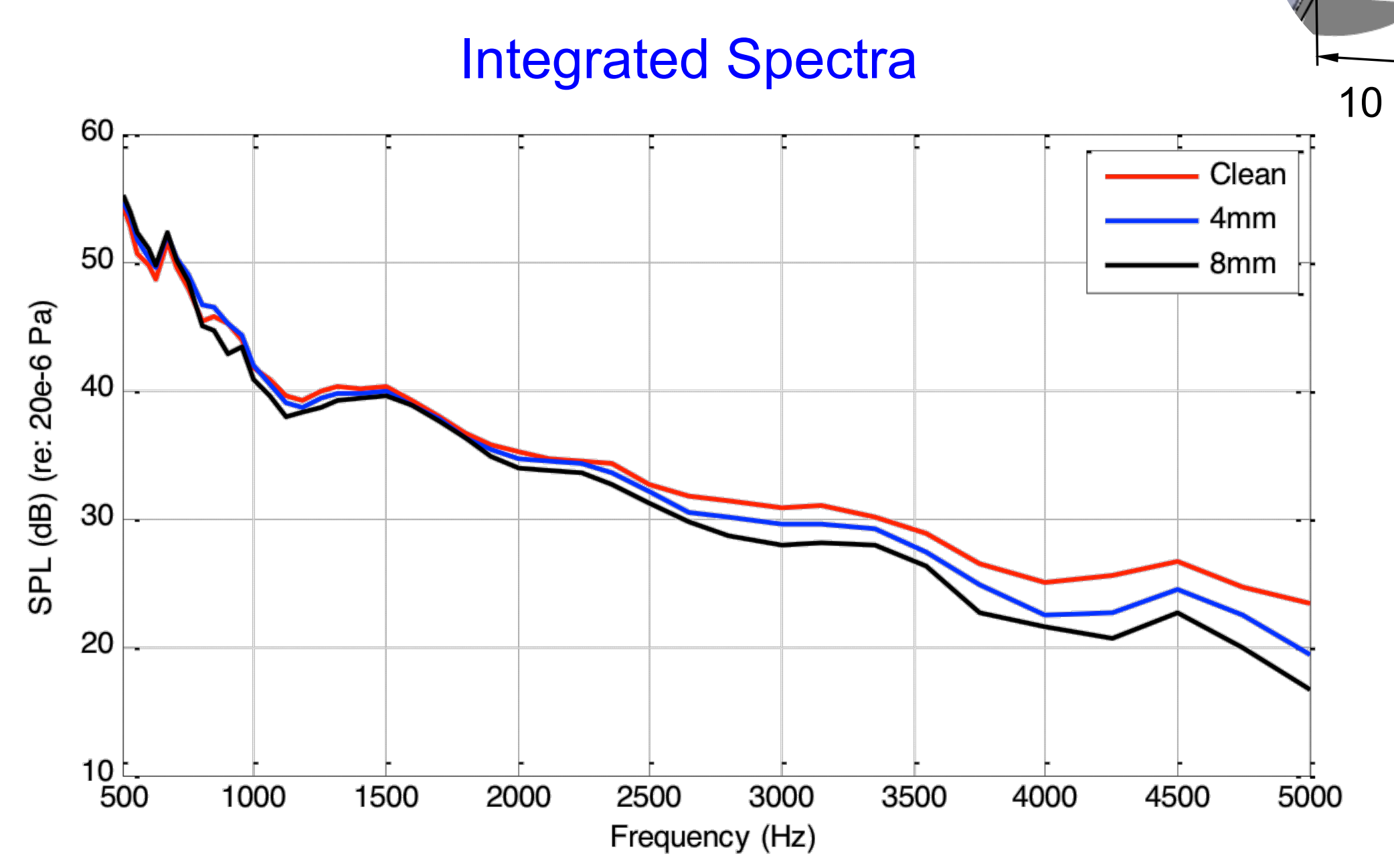

Thickness $-0.5 \mathrm{~mm}$ Spacing $-4 \mathrm{~mm}$ Height $-4,8 \mathrm{~mm}$ $10 \mathrm{~mm}$ Extension 


\section{Finlets - Effect of Extension}

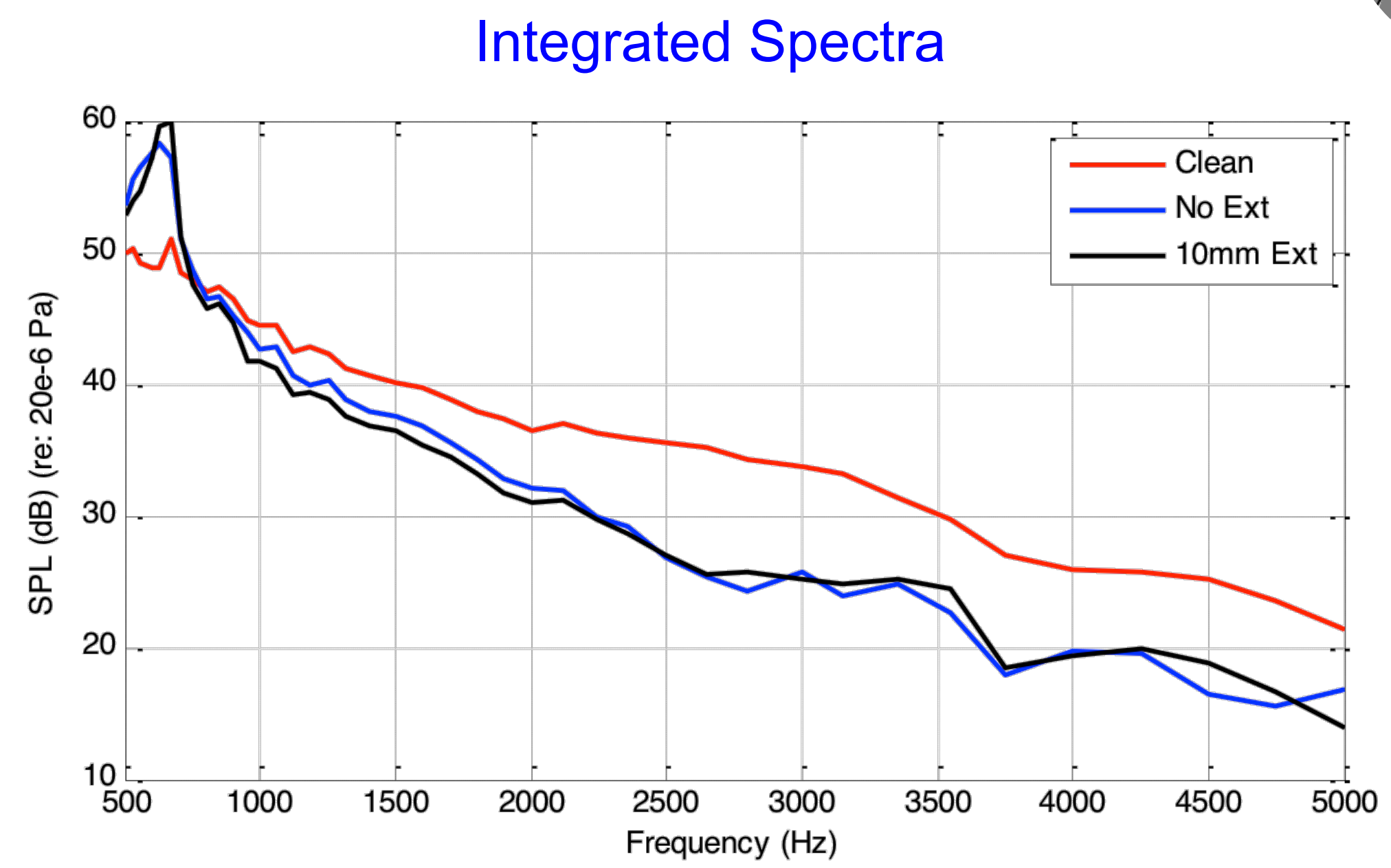

Thickness $-0.5 \mathrm{~mm}$

Spacing $-1 \mathrm{~mm}$

Height $-4 \mathrm{~mm}$

0,10mm Extension 


\section{Finlets - Effect of Extension}

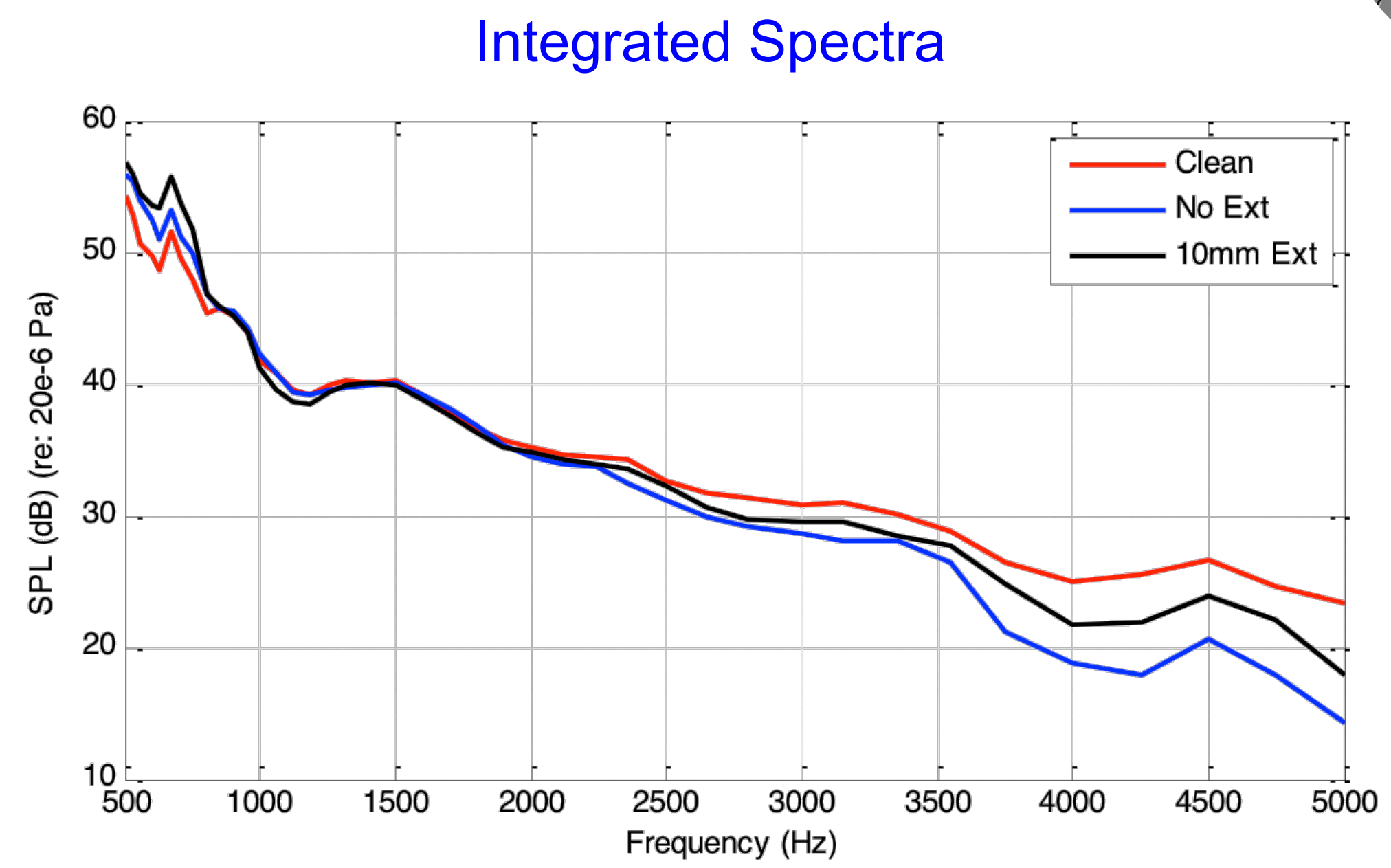

Thickness $-0.5 \mathrm{~mm}$

Spacing $-1 \mathrm{~mm}$

Height $-4 \mathrm{~mm}$

0,10mm Extension 


\section{Rails vs Finlets}

Integrated Spectra

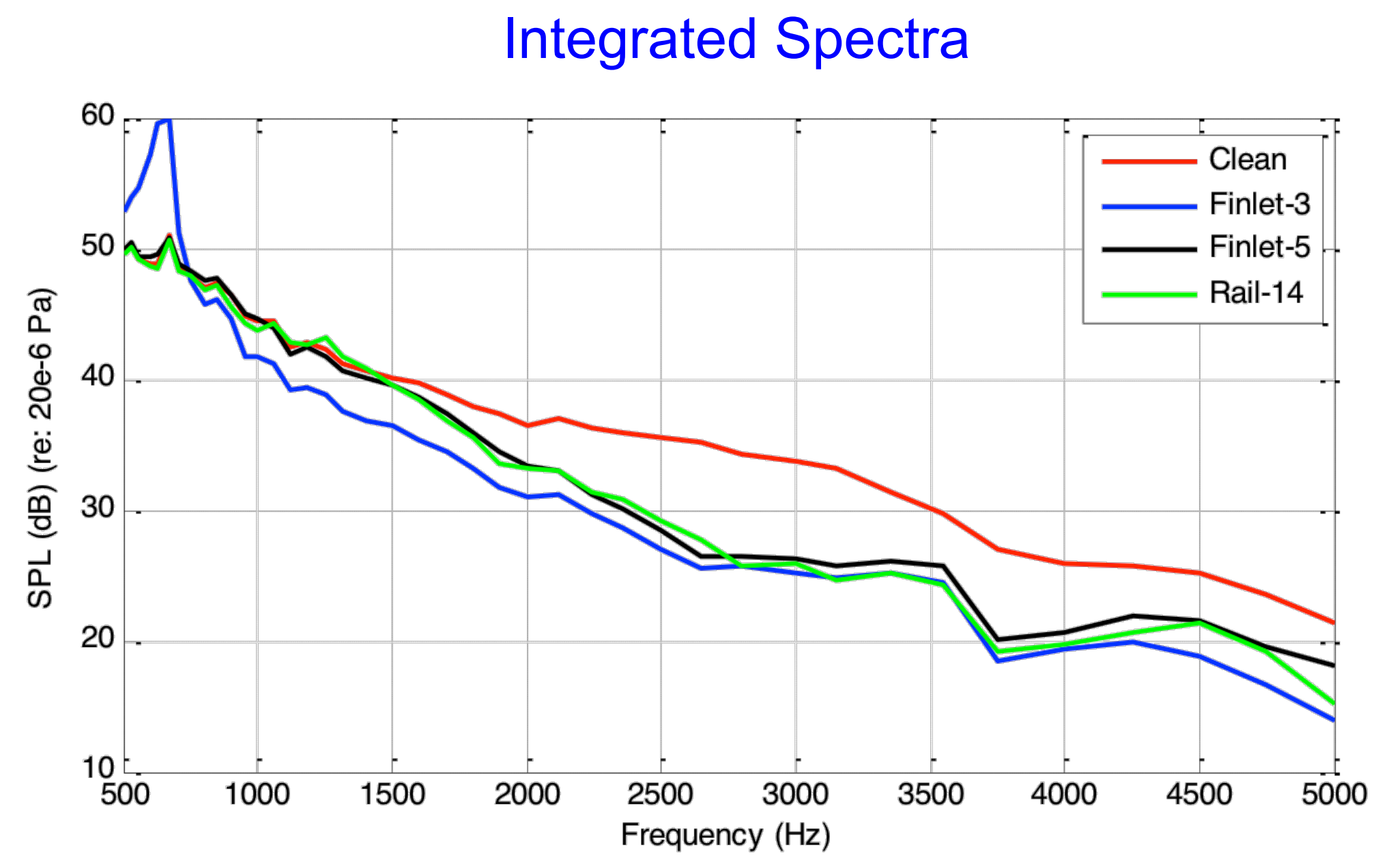

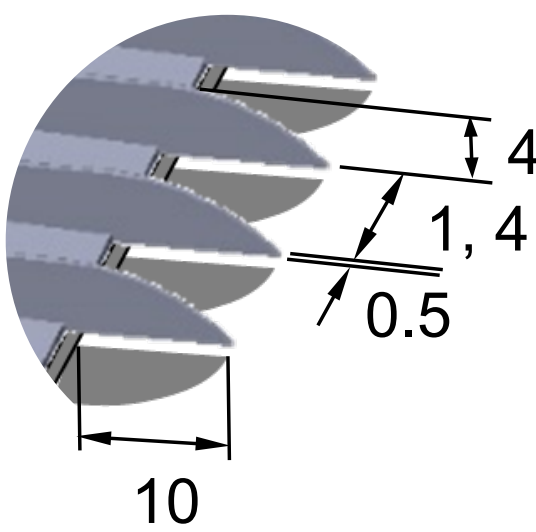

